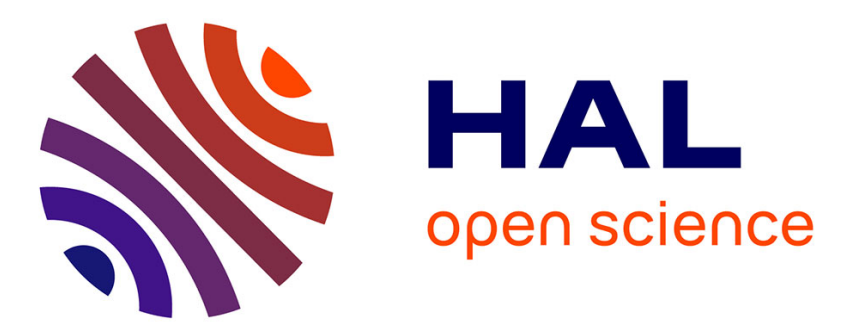

\title{
TWO ASYMPTOTIC MODELS FOR ARRAYS OF UNDERGROUND WASTE CONTAINERS
}

\author{
Grégoire Allaire, Marc Briane, Robert Brizzi, Yves Capdeboscq
}

\section{To cite this version:}

Grégoire Allaire, Marc Briane, Robert Brizzi, Yves Capdeboscq. TWO ASYMPTOTIC MODELS FOR ARRAYS OF UNDERGROUND WASTE CONTAINERS. Applicable Analysis, 2009, 88 (1011), pp.1445-1467. 10.1080/00036810902922590 . hal-00352797

\section{HAL Id: hal-00352797 https://hal.science/hal-00352797}

Submitted on 13 Jan 2009

HAL is a multi-disciplinary open access archive for the deposit and dissemination of scientific research documents, whether they are published or not. The documents may come from teaching and research institutions in France or abroad, or from public or private research centers.
L'archive ouverte pluridisciplinaire HAL, est destinée au dépôt et à la diffusion de documents scientifiques de niveau recherche, publiés ou non, émanant des établissements d'enseignement et de recherche français ou étrangers, des laboratoires publics ou privés. 


\title{
TWO ASYMPTOTIC MODELS FOR ARRAYS OF UNDERGROUND WASTE CONTAINERS
}

GRÉGOIRE ALLAIRE ${ }^{1}$, MARC BRIANE $^{2}$, ROBERT BRIZZI $^{1}$, YVES CAPDEBOSCQ $^{3}$

1 Centre de Mathématiques Appliquées, École Polytechnique, 91128 Palaiseau, France.

${ }^{2}$ Centre de Mathématiques, INSA de Rennes et IRMAR, 20 avenue des Buttes de Coësmes - 35043 Rennes, France.

${ }^{3}$ University of Oxford, OXFORD OX1 3LB, UK

\begin{abstract}
We study the homogenization of two models of an underground nuclear waste repository. The nuclear waste cells are periodically stored in the middle of a geological layer and are the only source terms in a parabolic evolution problem. The diffusion constants have a very large contrast between the fuel repository and the soil. It is thus a combined problem of homogenization and singular perturbation. For two different asymptotic contrasts we give the homogenized limit problem which is rigorously justified by using two-scale convergence. Eventually we perform 2-d numerical computations to show the effectiveness of using the limit model instead of the original one.
\end{abstract}

Key words: Homogenization, diffusion, porous media.

2000 Mathematics Subject Classification: 35B27, 74Q10.

\section{INTRODUCTION}

In this paper, we consider a simplified model used to describe the diffusion process of radioactive elements from a repository into surrounding geological layers. It is a parabolic evolution problem with very contrasted coefficients along a thin strip. Namely, we study the following model

(1)

$$
\left\{\begin{aligned}
\rho_{\varepsilon}(x) \frac{\partial u_{\varepsilon}}{\partial t}-\operatorname{div}\left(A_{\varepsilon}(x) \nabla u_{\varepsilon}\right)+\omega_{\varepsilon}(x) u_{\varepsilon} & =f_{\varepsilon}(t, x) \text { in } \Omega \text { for a.e. } t>0, \\
u_{\varepsilon} & =0 \text { on } \partial \Omega \text { for a.e. } t>0, \\
u_{\varepsilon}(t=0) & =0 \text { in } \Omega,
\end{aligned}\right.
$$

where $\Omega$ is a smooth open set of $\mathbb{R}^{d}$, with $d=2$ or 3 , which intersects the horizontal hyperplane passing through the origin, i.e., $\Omega \cup\left\{x_{d}=0\right\} \neq \emptyset$ (for any $x \in \mathbb{R}^{d}$ we write $x=\left(x^{\prime}, x_{d}\right)$ with $\left.x^{\prime} \in \mathbb{R}^{d-1}\right)$. The unknown is $u_{\varepsilon}(t, x) \in L^{2}\left(\mathbb{R}^{+} ; H_{0}^{1}(\Omega)\right)$. The repository, where the inhomogeneities and the source term $f_{\varepsilon}$ are located, is an horizontal strip of thickness $\varepsilon$ and midplane $\left\{x_{d}=0\right\}$. Furthermore, the small parameter $\varepsilon$ is the periodicity of the repository heterogeneities too. Outside the 


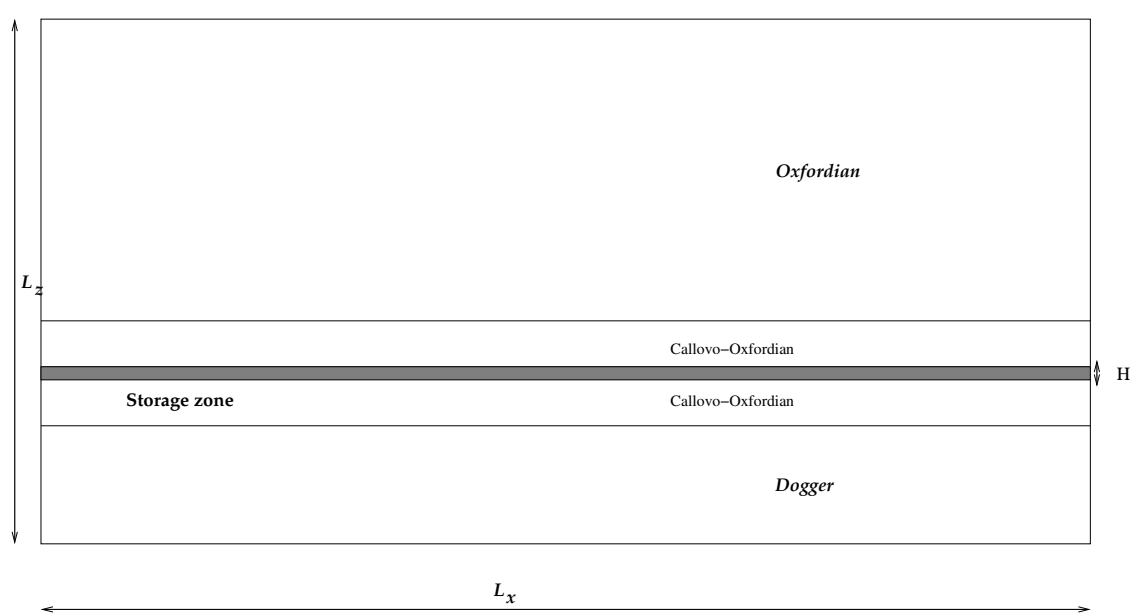

Figure 1. Sketch of the medium.

repository, the diffusion tensor $A_{\varepsilon}$ and the scalar porosities $\omega_{\varepsilon}$ and $\rho_{\varepsilon}$ are functions of the macroscopic or "slow" variable $x$ only. More precisely, we assume that there exist functions $\mu_{0}, \omega_{0}$ and $\rho_{0}$ in $L^{\infty}(\Omega)$ such that

$$
\left\{\begin{array}{l}
\text { for any } x=\left(x^{\prime}, x_{d}\right) \in \Omega \text { with }\left|x_{d}\right|>\varepsilon / 2, \quad f_{\varepsilon}(t, x)=0, \forall t \geq 0 \\
\text { and } A_{\varepsilon}(x)\left(\operatorname{resp} . \omega_{\varepsilon}(x), \rho_{\varepsilon}(x)\right)=\mu_{0}(x)\left(\operatorname{resp} . \omega_{0}(x), \rho_{0}(x)\right) .
\end{array}\right.
$$

Inside the repository, we assume that all coefficients, as well as the source term, are periodic in the first $(d-1)$ variables. We furthermore consider a highly contrasted diffusion tensor compared to $\mu_{0}$. Namely, we assume

(3)

$$
\left\{\begin{array}{l}
\text { for any } x=\left(x^{\prime}, x_{d}\right) \in \Omega \text { with }\left|x_{d}\right|<\varepsilon / 2, \quad f_{\varepsilon}(t, x)=\varepsilon^{-1} f_{1}\left(t, \frac{x}{\varepsilon}\right), \forall t \geq 0 \\
\text { and } A_{\varepsilon}(x)=\varepsilon^{a} \mu_{1}\left(\frac{x}{\varepsilon}\right), \omega_{\varepsilon}(x)=\omega_{1}\left(\frac{x}{\varepsilon}\right) \text { and } \rho_{\varepsilon}(x)=\rho_{1}\left(\frac{x}{\varepsilon}\right),
\end{array}\right.
$$

with periodic functions $\mu_{1} \in L_{\#, d}^{\infty}(Y)^{d \times d}, \omega_{1}, \rho_{1} \in L_{\#, d}^{\infty}(Y)$ and $f_{1} \in L^{\infty}\left(\mathbb{R}^{+} ; L_{\#, d}^{\infty}(Y)\right)$. We assume that $\omega_{0}, \rho_{0} \geq c>0$ (resp. $\omega_{1}, \rho_{1} \geq c>0$ ) where the constant $c$ is independent of $\varepsilon$ and that both diffusion tensors $\mu_{0}$ and $\mu_{1}$ are uniformly elliptic and bounded, that is, there exists two positive constants $\beta \geq \alpha>0$ such that, for all $\xi \in \mathbb{R}^{d}$,

$$
\alpha|\xi|^{2} \leq \mu_{i} \xi \cdot \xi \leq \beta|\xi|^{2} \quad i=0,1
$$

The exponent $a$ in (3) is either -1 or 2 . The periodicity cell is the unit cube $Y=(-1 / 2,+1 / 2)^{d}$, and by $L_{\#, d}^{\infty}(Y)$ we denote the following space

$$
\begin{gathered}
L_{\#, d}^{\infty}(Y)=\left\{f \in L^{\infty}\left(\mathbb{R}^{d}\right), \text { s.t. } \forall y=\left(y^{\prime}, y_{d}\right) \in \mathbb{R}^{d}\right. \\
\text { and } \left.\forall p \in \mathbb{Z}^{d-1}, f\left(y^{\prime}+p, y_{d}\right)=f\left(y^{\prime}, y_{d}\right)\right\} .
\end{gathered}
$$

Note that the scaling of the source term is such that its integral over the repository is of order one. We denote by $\chi_{\varepsilon}$ the characteristic function of the repository, that is, $\chi_{\varepsilon}(x)=\chi\left(\frac{x_{d}}{\varepsilon}\right)$ where $\chi$ is the indicator function of the interval $(-1 / 2,+1 / 2)$. We denote by $T>0$ the finite time-scale for which the model under consideration is valid. We also use the notations $\Omega^{\prime}=\Omega \cap\left\{x_{d}=0\right\}, \Omega^{+}=\Omega \cap\left\{x_{d}>0\right\}$, $\Omega^{-}=\Omega \cap\left\{x_{d}<0\right\}$, and $Y^{\prime}=(-1 / 2,+1 / 2)^{d-1}$. 
The motivation for this work is the study on the long time behavior of an underground waste repository in the presence of leakage. A sketch of the medium under consideration is presented in Figure 1. The spent fuel cells are stored in the center of the middle geological layer. Inside each module, 10 to 15 spent fuel cells are stored horizontally (normally to the section represented). A description of the geometry is presented in Section 6.1. This model problem, introduced as a benchmark case for the COUPLEX exercise [8], has been previously studied by Bourgeat et al. in [4], [9], [5], [6], [7]. The novelty here is that we allow a strong contrast between the fuel repository and the soil. The first case is that of a very small permeability of the engineered structure, $a=2$, where the repository is designed as a sealed containment area. The second case, is that of a large permeability, $a=-1$, where the repository structure is the weak point of the apparatus, the surrounding soil being a better natural barrier. This last case is what happens with a concrete structure is a clay layer. The source term represents a leakage (which would happen in these worst case scenario in every module).

The first part of the paper, sections 3 to 5 , is devoted to the asymptotic study of two models $(a=-1$ and $a=2)$ in the limit as $\varepsilon$ tends to zero. After establishing a priori estimates and recalling the notion of two scale convergence for boundary layers [3] in Section 3, we derive the homogenized limits for the case $a=2$ in Section 4 and for the case $a=-1$ in Section 5. A related result appeared in [14] with a different application to transmission through thin membrane.

The second part of the paper is dedicated to the application of this study in the nuclear repository context. The model used by the so-called MoMaS group ${ }^{1}$, in charge of the mathematical aspects of the long term underground waste repository behavior predictions, is slightly more complex than the one studied in the first part, and is exposed first. Because the coefficients are measured physical quantities, the relevant small parameter $\varepsilon$ has to be identified, and evaluated. With that in mind, we conduct a dimensional analysis of the available data, and express the MoMaS group model in this setting. The two cases we have considered correspond to limit situations, with very strong or very weak diffusion. The corresponding numerical results are presented in Section 6.

\section{Main Results}

The first result addresses the case when $a=2$, i.e. of very weak diffusion in the repository zone. In such a case, we need an additional technical assumption on the smoothness of the coefficients in the vicinity of the repository boundaries, namely

$$
\exists x_{0}^{d} \in(0,1 / 2) \text { and } r>0 \text { such that } \mu_{1}(y) \in C_{\#}^{0, r}\left(Y \cap\left\{\left|x_{d}\right|>x_{0}^{d}\right\}\right)^{d \times d} .
$$

Our result describes the limit behavior of $u_{\varepsilon}$ both outside the fuel repository and inside.

\footnotetext{
${ }^{1}$ The research group MoMaS (Mathematical Modeling and Numerical Simulation for Nuclear Waste Management Problems) is part of the PACE Research Federation. MoMaS's sponsors are: ANDRA National Radioactive Waste Management Agency, BRGM Bureau des recherches géologiques et minières, CEA Atomic Energy Commission, CNRS National Center for Scientific Research, EDF Électricité de France, IRSN Institute for Radiological Protection and Nuclear Safety.
} 
Theorem 1. Under assumptions (2,3,4,5), when $a=2$, the solution $u_{\varepsilon}$ of (1) is such that $\left(1-\chi_{\varepsilon}\right) u_{\varepsilon}$ converges strongly in $L^{2}\left((0, T) ; L^{2}(\Omega)\right)$ to $u$, given by $u:=u^{+}$ in $\Omega^{+}$and $u:=u^{-}$in $\Omega^{-}$, and $\left(1-\chi_{\varepsilon}\right) \nabla u_{\varepsilon}$ converges weakly in $L^{2}\left((0, T) ; L^{2}(\Omega)^{d}\right)$ to $\xi$, with $\xi=\nabla u^{ \pm}$in $\Omega^{ \pm}$, where $u^{ \pm}$is the unique solution of

$$
\left\{\begin{aligned}
\rho_{0}(x) \frac{\partial u^{ \pm}}{\partial t}-\operatorname{div}\left(\mu_{0}(x) \nabla u^{ \pm}\right)+\omega_{0}(x) u^{ \pm}=0 & \text { in } \Omega^{ \pm}, \\
u^{ \pm}=0 & \text { on } \partial \Omega \cap \partial \Omega^{ \pm}, \\
\mp \mu_{0}(x) \nabla u^{ \pm} \cdot e_{d}=s^{ \pm} & \text {on } \Omega^{\prime}, \\
u^{ \pm}(t=0)=0 & \text { in } \Omega .
\end{aligned}\right.
$$

The effective source terms $s^{+}$and $s^{-}$are given by

$$
s^{ \pm}(t)= \pm \int_{Y^{\prime}} \mu_{1}\left(y^{\prime}, \pm 1 / 2\right) \nabla u_{2}\left(t, y^{\prime}, \pm 1 / 2\right) \cdot e_{d} d y^{\prime},
$$

where $u_{2}$ is the limit of $\varepsilon u_{\varepsilon}$ in the sense of two-scale boundary layers convergence (see Proposition 5). The limit $u_{2}(t, y)$ is the unique solution in $L^{2}\left((0, T) ; H^{1}(Y)\right)$ of the following problem

$$
\left\{\begin{aligned}
\rho_{1}(y) \frac{\partial u_{2}}{\partial t}-\operatorname{div}_{y}\left(\mu_{1}(y) \nabla_{y} u_{2}\right)+\omega_{1}(y) u_{2} & =f_{1}(t, y) \text { in }(0, T) \times Y, \\
u_{2}(t, y) & =0 \text { on }(0, T) \times\left\{\left|y_{d}\right|=1 / 2\right\}, \\
\left(y_{1}, \ldots, y_{d-1}\right) & \rightarrow u_{2}(t, y) Y^{\prime} \text {-periodic, } \\
u_{2}(t=0) & =0 .
\end{aligned}\right.
$$

Asymptotically, the effect of the strip is therefore to transform the source term $f_{1}$ into two effective time-dependent boundary conditions $s^{+}$or $s^{-}$for the normal derivatives given by (7). Note that system (8) is independent of system (6). Such a limit problem is the inspiration for an approximate numerical method presented in Section 6. Theorem 1 is related to a recent result in [14], for nonlinear reactiondiffusion systems. However the scaling, as well as the analysis, are quite different.

We now address the second case when $a=-1$.

Theorem 2. Under assumptions (2,3,4), when $a=-1$, the solution $u_{\varepsilon}$ of (1) is such that it converges strongly in $L^{2}\left((0, T) ; L^{2}(\Omega)\right)$ to $u$ and $\nabla u_{\varepsilon}$ converges weakly in $L^{2}\left((0, T) ; L^{2}(\Omega)^{d}\right)$ to $\nabla u$, where $u \in H^{1}(\Omega)$ is the unique solution of the coupled system

$$
\left\{\begin{aligned}
\rho_{0} \frac{\partial u}{\partial t}-\operatorname{div}\left(\mu_{0} \nabla u\right)+\omega_{0} u & =0 \text { in } \Omega^{+} \cup \Omega^{-} \\
u(t=0) & =0 \text { in } \Omega^{+} \cup \Omega^{-} \\
u & =0 \text { on } \partial \Omega \\
u & =u_{-1} \text { on } \Omega^{\prime}=\Omega \cap\left\{x_{d}=0\right\}
\end{aligned}\right.
$$

and $u_{-1}$ is the solution in $H^{1}\left(\Omega^{\prime}\right)$ of

$$
-\operatorname{div}_{x^{\prime}}\left(\mu^{*} \nabla_{x^{\prime}} u_{-1}(t, x)\right)+\left[\mu_{0} \nabla u \cdot n\right]=\bar{f}
$$


where $[j]$ stands for the jump of a quantity $j$ between $\Omega^{+}$and $\Omega^{-}$. The effective coefficients $\mu^{*}$ and $\bar{f}$ are given by

$$
\bar{f}(t)=\int_{Y} f_{1}(t, y) d y
$$

and

$$
\mu^{*}=\int_{Y} \mu_{1}(y)\left(I_{d}+P(y)\right) d y
$$

For each $i=1, \ldots, d, P(\cdot) e_{i}$ is given by $P(\cdot) e_{i}=\nabla \varphi_{i}$ where $\varphi_{i} \in H_{\#, d}^{1}(Y)$ is $Y^{\prime}$-periodic in its $(d-1)$ first variables and is the unique solution, up to an additive constant, of

$$
\left\{\begin{array}{l}
\operatorname{div}\left(\mu_{1}\left(\nabla y_{i}+\nabla_{y} \varphi_{i}\right)\right)=0 \text { in } Y, \\
\mu_{1}\left(\nabla y_{i}+\nabla_{y} \varphi_{i}\right) \cdot e_{d}=0 \text { on } Y^{\prime} \times\{+1 / 2\}, \\
\mu_{1}\left(\nabla y_{i}+\nabla_{y} \varphi_{i}\right) \cdot e_{d}=0 \text { on } Y^{\prime} \times\{-1 / 2\} .
\end{array}\right.
$$

Of course, the two partial differential equations (9) and (10) are strongly coupled. Asymptotically, the effect of the strip is therefore to transform the source term $f_{1}$ into an effective time-dependent tangential diffusion along the interface given by (10). The homogenized problem (9)-(10) is the inspiration for an approximate numerical method in Section 6. For a homogeneous thin strip, Theorem 2 was already obtained in [12].

\section{A PRIORI ESTIMATES AND TWO-SCALE BOUNDARY LAYER CONVERGENCE}

A prerequisite for the proofs of Theorems 1 and 2 is to check that the solution $u_{\varepsilon}$ of (1) is uniformly bounded in appropriate norms. The necessary a priori estimates are established in the following Proposition.

Proposition 3. Assume the coefficients $A_{\varepsilon}, \omega_{\varepsilon}, \rho_{\varepsilon}$ satisfy assumptions (2, 3, 4).

(i) If, instead of assumption (3), we suppose that $f_{\varepsilon}(t, x)=\varepsilon^{b} \chi_{\varepsilon}\left(\frac{x_{d}}{\varepsilon}\right) f_{1}\left(t, \frac{x}{\varepsilon}\right)$ with $f_{1} \in L^{\infty}\left(\mathbb{R}^{+} ; L_{\#, d}^{\infty}\left(Y^{\prime} \times(-1 / 2,1 / 2)\right)\right)$, and $b \in \mathbb{R}$, we have

$$
\left\|u_{\varepsilon}\right\|_{L^{\infty}\left((0, T) ; L^{2}(\Omega)\right)}+\left\|\left(1-\chi_{\varepsilon}\right) \nabla u_{\varepsilon}\right\|_{L^{2}\left((0, T) ; L^{2}(\Omega)^{d}\right)}
$$

$$
+\varepsilon^{\frac{a}{2}}\left\|\chi_{\varepsilon} \nabla u_{\varepsilon}\right\|_{L^{2}\left((0, T) ; L^{2}(\Omega)^{d}\right)} \leq C \varepsilon^{\frac{1}{2}+b} \min \left(1, \varepsilon^{-\frac{a}{2}}\right),
$$

(ii) Alternatively, instead of assumption (3) assume that $f_{\varepsilon}(t, x)=s_{\varepsilon}\left(t, x^{\prime}\right) \delta_{\left\{\left|x_{d}\right|=\varepsilon / 2\right\}}\left(\frac{x_{d}}{\varepsilon}\right)$ where $s_{\varepsilon} \in C\left(\mathbb{R}^{+} \times \mathbb{R}^{d-1}\right)$ is bounded above and below independently of $\varepsilon$. Then we have

$\left\|u_{\varepsilon}\right\|_{L^{\infty}\left(0, T, L^{2}(\Omega)\right)}+\left\|\left(1-\chi_{\varepsilon}\right) \nabla u_{\varepsilon}\right\|_{L^{2}\left((0, T) ; L^{2}(\Omega)^{d}\right)}+\varepsilon^{\frac{a}{2}}\left\|\chi_{\varepsilon} \nabla u_{\varepsilon}\right\|_{L^{2}\left((0, T) ; L^{2}(\Omega)^{d}\right)} \leq C$.

Corollary 4. Assume the coefficients $A_{\varepsilon}, \omega_{\varepsilon}, \rho_{\varepsilon}$ satisfy assumptions (2, 3 and 4). If $a=-1$, then we have

$$
\left\|u_{\varepsilon}\right\|_{L^{\infty}\left((0, T) ; L^{2}(\Omega)\right)}+\left\|\left(1-\chi_{\varepsilon}\right) \nabla u_{\varepsilon}\right\|_{L^{\infty}\left((0, T) ; L^{2}(\Omega)^{d}\right)} \leq C,
$$

and

$$
\left\|\chi_{\varepsilon} u_{\varepsilon}\right\|_{L^{2}\left((0, T) ; L^{2}(\Omega)\right)}+\left\|\chi_{\varepsilon} \nabla u_{\varepsilon}\right\|_{L^{2}\left((0, T) ; L^{2}(\Omega)^{d}\right)} \leq C \sqrt{\varepsilon}
$$


If $a=2$, then $w_{\varepsilon}=\varepsilon u_{\varepsilon}$ satisfies

(18)

$\left\|w_{\varepsilon}\right\|_{L^{\infty}\left((0, T) ; L^{2}(\Omega)\right)}+\left\|\left(1-\chi_{\varepsilon}\right) \nabla w_{\varepsilon}\right\|_{L^{2}\left((0, T) ; L^{2}(\Omega)^{d}\right)}+\varepsilon\left\|\chi_{\varepsilon} \nabla w_{\varepsilon}\right\|_{L^{2}\left((0, T) ; L^{2}(\Omega)^{d}\right)} \leq C \sqrt{\varepsilon}$

which, in particular, implies that

$$
\left\|\chi_{\varepsilon} w_{\varepsilon}\right\|_{L^{\infty}\left((0, T) ; L^{2}(\Omega)\right)}+\varepsilon\left\|\chi_{\varepsilon} \nabla w_{\varepsilon}\right\|_{L^{2}\left((0, T) ; L^{2}(\Omega)^{d}\right)} \leq C \sqrt{\varepsilon} .
$$

Proof of Corollary 4. Estimates (16), (18) and (19) are obvious consequences of Proposition 3. Inequality (17) is easily deduced from (14) and (22).

Proof of Proposition 3. Testing (1) against $u_{\varepsilon}$ we obtain, after integration by parts with respect to the space variable $x$,

$$
\begin{aligned}
\frac{1}{2} \frac{d}{d t} \int_{\Omega} \rho_{\varepsilon} u_{\varepsilon}^{2} d x+\int_{\Omega}\left(1-\chi_{\varepsilon}\right) \mu_{0}(x) & \nabla u_{\varepsilon} \cdot \nabla u_{\varepsilon} d x+\int_{\Omega} \omega\left(\frac{x}{\varepsilon}\right) u_{\varepsilon}^{2} d x \\
& +\varepsilon^{a} \int_{\Omega} \chi_{\varepsilon} \mu_{1}\left(\frac{x}{\varepsilon}\right) \nabla u_{\varepsilon} \cdot \nabla u_{\varepsilon} d x
\end{aligned}
$$

Let us first address the case (i): there is a constant $M$ such that $\left\|f_{\varepsilon}\right\|_{L^{\infty}\left(\mathbb{R}^{+} \times \Omega\right)} \varepsilon^{-b}<$ $M<\infty$ for any $\varepsilon$. Then (20) yields, because of the positivity of $\rho_{\varepsilon}, \omega_{\varepsilon}$ and the coercivity of $\mu_{0}$ and $\mu_{1}$, that

$$
\frac{d}{d t}\left\|\rho_{\varepsilon}^{\frac{1}{2}} u_{\varepsilon}\right\|_{L^{2}(\Omega)}^{2} \leq C\left\|\chi_{\varepsilon} f_{\varepsilon}\right\|_{L^{2}(\Omega)}\left\|\chi_{\varepsilon} \rho_{\varepsilon}^{\frac{1}{2}} u_{\varepsilon}\right\|_{L^{2}(\Omega)} \leq C \varepsilon^{\frac{1}{2}+b}\left\|\chi_{\varepsilon} \rho_{\varepsilon}^{\frac{1}{2}} u_{\varepsilon}\right\|_{L^{2}(\Omega)},
$$

which in turns shows that $\left\|u_{\varepsilon}(t, \cdot)\right\|_{L^{2}(\Omega)} \leq C t \varepsilon^{\frac{1}{2}+b}$, the constant $C$, depending a priori on $\min _{\Omega}\left|\rho_{\varepsilon}\right|$, and $M$ being chosen independently of $\varepsilon$. Integrating (20) with respect to time, and using Cauchy-Schwarz inequality again we obtain,

$$
\left\|\left(1-\chi_{\varepsilon}\right) \nabla u_{\varepsilon}\right\|_{L^{2}\left((0, T) ; L^{2}(\Omega)^{d}\right)}^{2}+\varepsilon^{a}\left\|\chi_{\varepsilon} \nabla u_{\varepsilon}\right\|_{L^{2}\left((0, T) ; L^{2}(\Omega)^{d}\right)}^{2} \leq C \varepsilon^{1+2 b} .
$$

This implies (14). On the other hand, thanks to the Dirichlet boundary condition satisfied by $u_{\varepsilon}$ in (1), we have, for all $t>0$, the following Poincaré inequality

$$
\left\|\chi_{\varepsilon} u_{\varepsilon}\right\|_{L^{2}(\Omega)}^{2} \leq d_{\Omega}\left\|\chi_{\varepsilon} \nabla u_{\varepsilon}\right\|_{L^{2}(\Omega)^{d}}^{2},
$$

where $d_{\Omega}$ depends on $\Omega$ only. This can be proved, for example, by integrating $u_{\varepsilon}$ along a vertical or horizontal path connecting $x$ and the boundary of the domain within the repository. Consequently, we have, for all $t>0$, and all $\lambda>0$,

$$
\begin{aligned}
\int_{\Omega} \chi_{\varepsilon} f_{\varepsilon} u_{\varepsilon} d x & \leq \varepsilon^{-a} \frac{d_{\Omega}}{4 \lambda \alpha}\left\|\chi_{\varepsilon} f_{\varepsilon}\right\|_{L^{2}(\Omega)}^{2}+\varepsilon^{a} \frac{\alpha \lambda}{d_{\Omega}}\left\|\chi_{\varepsilon} u_{\varepsilon}\right\|_{L^{2}(\Omega)}^{2} \\
& \leq \varepsilon^{-a} \frac{d_{\Omega}}{4 \alpha \lambda}\left\|\chi_{\varepsilon} f_{\varepsilon}\right\|_{L^{2}(\Omega)}^{2}+\varepsilon^{a} \lambda \int_{\Omega} \chi_{\varepsilon} \mu_{1}\left(\frac{x}{\varepsilon}\right) \nabla u_{\varepsilon} \cdot \nabla u_{\varepsilon} d x
\end{aligned}
$$

For $\lambda=1$, this last inequality combined with (20) yields

$$
\begin{aligned}
\frac{1}{2} \frac{d}{d t} \int_{\Omega} \rho_{\varepsilon} u_{\varepsilon}^{2} d x & \leq \frac{d_{\Omega}}{4 \alpha} \varepsilon^{-a}\left\|\chi_{\varepsilon} f_{\varepsilon}\right\|_{L^{2}(\Omega)}^{2} \\
& \leq C \varepsilon^{-a+1+2 b},
\end{aligned}
$$


which shows that $\left\|u_{\varepsilon}(t, \cdot)\right\|_{L^{2}(\Omega)} \leq C \varepsilon^{\frac{1}{2}+b-\frac{1}{2} a}$. For $\lambda=1 / 2$, using (23) in (20) we obtain that for all $t>0$,

$$
\begin{aligned}
\frac{1}{2} \frac{d}{d t} \int_{\Omega} \rho_{\varepsilon} u_{\varepsilon}^{2} d x & +\int_{\Omega}\left(1-\chi_{\varepsilon}\right) \mu_{0}(x) \nabla u_{\varepsilon} \cdot \nabla u_{\varepsilon} d x \\
& +\frac{1}{2} \varepsilon^{a} \int_{\Omega} \chi_{\varepsilon} \mu_{1}\left(\frac{x}{\varepsilon}\right) \nabla u_{\varepsilon} \cdot \nabla u_{\varepsilon} d x \leq C \varepsilon^{-a+1+2 b},
\end{aligned}
$$

which in turn implies that

$$
\left\|\left(1-\chi_{\varepsilon}\right) \nabla u_{\varepsilon}\right\|_{L^{2}\left((0, T) ; L^{2}(\Omega)^{d}\right)}^{2}+\varepsilon^{a}\left\|\chi_{\varepsilon} \nabla u_{\varepsilon}\right\|_{L^{2}\left((0, T) ; L^{2}(\Omega)^{d}\right)}^{2} \leq C \varepsilon^{-a+1+2 b} .
$$

Estimate (14) is proved, and the proof of the first part of Proposition 3 is finished.

Let us now turn to case (ii), and suppose that $f_{\varepsilon}=s_{\varepsilon}\left(t, x^{\prime}\right) \delta_{\left\{\left|x_{d}\right|=\varepsilon / 2\right\}}$ with $s_{\varepsilon} \in C\left(\mathbb{R}^{+} \times \mathbb{R}^{d-1}\right)$, and $\left\|s_{\varepsilon}\right\|_{L^{\infty}}<M$ where the constant $M$ is independent of $\varepsilon$. Then

$$
\begin{aligned}
(25) \int_{\Omega} f_{\varepsilon}(t, x) u_{\varepsilon} d x & \leq M \int_{\left\{x_{d}= \pm \varepsilon / 2\right\}}\left|u_{\varepsilon}\right| d x^{\prime} \leq \frac{M^{2}}{\tau}+C \tau \int_{\left\{x_{d}= \pm \varepsilon / 2\right\}}\left|u_{\varepsilon}\right|^{2} d x^{\prime} \\
& \leq \frac{M^{2}}{\tau}+\int_{\Omega}\left(1-\chi_{\varepsilon}\right) \mu_{0}(x) \nabla u_{\varepsilon} \cdot \nabla u_{\varepsilon} d x
\end{aligned}
$$

for a sufficiently small constant $\tau>0$. Therefore we obtain

$$
\frac{1}{2} \frac{d}{d t} \int_{\Omega} \rho_{\varepsilon}(x) u_{\varepsilon}^{2} d x \leq \frac{M^{2}}{\tau},
$$

and arguing as above, we obtain (15).

Following the strategy introduced in [3], we shall derive the asymptotic limit of problem (1) using two-scale convergence for boundary layers. This notion of convergence generalizes the usual two-scale convergence [2], [13] to the case when the test functions periodically oscillate in the first $(d-1)$ space directions and simply decay at infinity in the last direction. The following proposition summarizes the definition and properties of two-scale convergence in the sense of boundary layers. We denote by $G$ the band $Y^{\prime} \times \mathbb{R}$. A generic point $y \in G$ is denoted by $y=\left(y^{\prime}, y_{d}\right)$ with $y^{\prime} \in Y^{\prime}$ and $y_{d} \in \mathbb{R}$. We introduce the space $L_{\#}^{2}(G)$ of square integrable functions on $G$ which are periodic in the first $d-1$ variables, i.e.

$$
L_{\#}^{2}(G)=\left\{\varphi \in L^{2}(G) \text { s.t. } y^{\prime} \mapsto \varphi\left(y^{\prime}, y_{d}\right) \text { is } Y^{\prime} \text {-periodic }\right\} \text {. }
$$

Similarly, we define $H_{\#}^{1}(G)$ the Sobolev space of functions in $H^{1}(G)$ which are $Y^{\prime}$ periodic, and $C_{\#, c}^{\infty}(G)$ and $C_{\#}^{\infty}(G)$ the space of infinitely differentiable functions -with compact support in the first case- on $G$. We denote by $C\left(\overline{\Omega^{\prime}}\right)$ the space of continuous functions on the closure of $\Omega^{\prime}$, a compact set in $\mathbb{R}^{d-1}$.

Proposition 5. (1) Let $v_{\varepsilon}$ be a sequence in $L^{2}(\Omega)$ such that

$$
\left\|v_{\varepsilon}\right\|_{L^{2}(\Omega)} \leq C \sqrt{\varepsilon} .
$$

There exists a subsequence, still denoted by $\varepsilon$, and a limit $v_{0}\left(x^{\prime}, y\right) \in L^{2}\left(\Omega^{\prime} ; L_{\#}^{2}(G)\right)$ such that $v_{\varepsilon}$ two-scale converges weakly in the sense of boundary layers to $v_{0}$ i.e.

$$
\lim _{\varepsilon \rightarrow 0} \frac{1}{\varepsilon} \int_{\Omega} v_{\varepsilon}(x) \varphi\left(x^{\prime}, \frac{x}{\varepsilon}\right) d x=\frac{1}{\left|Y^{\prime}\right|} \int_{\Omega^{\prime}} \int_{G} v_{0}\left(x^{\prime}, y\right) \varphi\left(x^{\prime}, y\right) d x^{\prime} d y
$$


for all test functions $\varphi\left(x^{\prime}, y\right) \in C\left(\overline{\Omega^{\prime}} ; L_{\#}^{2}(G)\right)$.

(2) Let $v_{\varepsilon}$ be a sequence which two-scale converges weakly in the sense of boundary layers to $v_{0}$, and furthermore satisfies

$$
\lim _{\varepsilon \rightarrow 0} \frac{1}{\sqrt{\varepsilon}}\left\|v_{\varepsilon}\right\|_{L^{2}(\Omega)}=\frac{1}{\left|Y^{\prime}\right|^{\frac{1}{2}}}\left\|v_{0}\right\|_{L^{2}\left(\Omega^{\prime} \times G\right)} .
$$

Then, $v_{\varepsilon}$ is said to two-scale converge strongly in the sense of boundary layers to $v_{0}$, which means that, for any sequence $w_{\varepsilon}$ in $L^{2}(\Omega)$ which two-scale converges weakly in the sense of boundary layers to $w_{0}\left(x^{\prime}, y\right) \in L^{2}\left(\Omega^{\prime} \times G\right)$ one has

$$
\lim _{\varepsilon \rightarrow 0} \frac{1}{\varepsilon} \int_{\Omega} v_{\varepsilon}(x) w_{\varepsilon}(x) \varphi\left(x^{\prime}, \frac{x}{\varepsilon}\right) d x=\frac{1}{\left|Y^{\prime}\right|^{\frac{1}{2}}} \int_{\Omega^{\prime}} \int_{G} v_{0}\left(x^{\prime}, y\right) w_{0}\left(x^{\prime}, y\right) \varphi\left(x^{\prime}, y\right) d x^{\prime} d y,
$$

for all smooth functions $\varphi\left(x^{\prime}, y\right) \in C\left(\overline{\Omega^{\prime}} ; C_{\#, c}(G)\right)$.

Note that two-scale boundary layer convergence (called 2SBL in the sequel) can be also used to characterize the precise behavior of a non-vanishing function, restricted to a small domain, as it is shown by the following simple lemma.

Lemma 6. Let $v_{\varepsilon}$ be a bounded sequence in $H_{0}^{1}(\Omega)$ converging weakly to a limit $v$. Assume that $w_{\varepsilon}=\chi_{\varepsilon} v_{\varepsilon}$ admits a limit $w$ in the sense of boundary layers convergence. Then $w=\chi w$ and for almost every $x^{\prime} \in \Omega^{\prime}$,

$$
v\left(x^{\prime}, 0\right)=\int_{Y} w\left(x^{\prime}, y\right) d y
$$

Proof. Recall that $\chi_{\varepsilon}=\chi\left(\frac{x}{\varepsilon}\right)$ is the characteristic function of the repository zone. The fact that $w=\chi w$ is obvious. Note that the assumption on $w_{\varepsilon}$ having a twoscale limit in the sense of boundary layers is plausible since, if $v_{\varepsilon}$ is a constant sequence $v \in L^{\infty}(\Omega)$, then $w_{\varepsilon}$ satisfies the a priori estimate $\left\|w_{\varepsilon}\right\|_{L^{2}(\Omega)} \leq C \sqrt{\varepsilon}$ which is required for the two-scale convergence in the sense of boundary layers. We have

$$
\begin{aligned}
& \frac{1}{\varepsilon} \int_{\Omega} \chi_{\varepsilon} v_{\varepsilon}\left(x^{\prime}, 0\right) \varphi(x) d x-\int_{\Omega^{\prime}} v_{\varepsilon}\left(x^{\prime}, 0\right) \varphi\left(x^{\prime}, 0\right) d x^{\prime} \\
= & \frac{1}{\varepsilon} \int_{\Omega} \chi_{\varepsilon} v_{\varepsilon}\left(x^{\prime}, 0\right) \varphi\left(x^{\prime}, 0\right) d x-\int_{\Omega^{\prime}} v_{\varepsilon}\left(x^{\prime}, 0\right) \varphi\left(x^{\prime}, 0\right) d x^{\prime} \\
+ & \int_{\Omega} v_{\varepsilon}\left(x^{\prime}, 0\right) \frac{1}{\varepsilon} \chi_{\varepsilon}\left(\varphi\left(x^{\prime}, 0\right)-\varphi(x)\right) d x .
\end{aligned}
$$

The first term is exactly zero. For the second one, note that

$$
\left|\frac{1}{\varepsilon} \chi_{\varepsilon}\left(\varphi\left(x^{\prime}, 0\right)-\varphi(x)\right)\right| \leq \frac{1}{\varepsilon} \int_{-\varepsilon / 2}^{\varepsilon / 2}\left|\frac{\partial}{\partial x_{d}} \varphi\left(x^{\prime}, t\right)\right| d t \leq\|\nabla \varphi\|_{L^{\infty}(\Omega)},
$$

therefore,

$$
\begin{aligned}
\left|\int_{\Omega} v_{\varepsilon}\left(x^{\prime}, 0\right) \frac{1}{\varepsilon} \chi_{\varepsilon}\left(\varphi\left(x^{\prime}, 0\right)-\varphi(x)\right) d x\right| & \leq\|\nabla \varphi\|_{L^{\infty}(\Omega)} \varepsilon \int_{\Omega^{\prime}} v_{\varepsilon}\left(x^{\prime}, 0\right) d x^{\prime} \\
& \leq C \varepsilon\|\nabla \varphi\|_{L^{\infty}(\Omega)}\left\|\nabla v^{\varepsilon}\right\|_{L^{2}(\Omega)^{d}}
\end{aligned}
$$


TWO ASYMPTOTIC MODELS FOR ARRAYS OF UNDERGROUND WASTE CONTAINERS 9

where this last term is of order $\mathcal{O}(\varepsilon)$ since the sequence $v_{\varepsilon}$ is bounded in $H_{0}^{1}(\Omega)$. Similarly,

$$
\begin{aligned}
\int_{\Omega}\left|\frac{1}{\varepsilon} \chi_{\varepsilon}\left(v_{\varepsilon}\left(x^{\prime}, 0\right)-v_{\varepsilon}(x)\right)\right| d x & \leq \int_{\Omega} \frac{\chi_{\varepsilon}}{\varepsilon} \int_{-\varepsilon / 2}^{\varepsilon / 2}\left|\frac{\partial}{\partial x_{d}} v_{\varepsilon}\left(x^{\prime}, s_{d}\right)\right| d s_{d} d x \\
& \leq C \sqrt{\varepsilon}\left\|\nabla v^{\varepsilon}\right\|_{L^{2}(\Omega)^{d}} .
\end{aligned}
$$

As a consequence,

$$
\begin{aligned}
& \left|\int_{\Omega} \frac{1}{\varepsilon} \chi_{\varepsilon} v_{\varepsilon(x)} \varphi(x) d x-\int_{\Omega^{\prime}} v\left(x^{\prime}, 0\right) \varphi\left(x^{\prime}, 0\right) d x^{\prime}\right| \\
\leq & \left|\int_{\Omega} \frac{1}{\varepsilon} \chi_{\varepsilon} v_{\varepsilon}\left(x^{\prime}, 0\right) \varphi(x) d x-\int_{\Omega^{\prime}} v\left(x^{\prime}, 0\right) \varphi\left(x^{\prime}, 0\right) d x^{\prime}\right|+\mathcal{O}(\sqrt{\varepsilon}) \\
\leq & \int_{\Omega^{\prime}}\left|v_{\varepsilon}\left(x^{\prime}, 0\right)-v\left(x^{\prime}, 0\right)\right|\left|\varphi\left(x^{\prime}, 0\right)\right| d x^{\prime}+\mathcal{O}(\sqrt{\varepsilon}) \\
\leq & C(\varphi)\left\|v_{\varepsilon}(\cdot, 0)-u(\cdot, 0)\right\|_{L^{2}\left(\Omega^{\prime}\right)}+\mathcal{O}(\sqrt{\varepsilon}) .
\end{aligned}
$$

The trace of $v_{\varepsilon}$ on $\Omega^{\prime}$ converge strongly in $L^{2}\left(\Omega^{\prime}\right)$ to the trace of $v$, thus we have proved that

$$
\begin{aligned}
\int_{\Omega^{\prime}} \int_{G} w\left(x^{\prime}, y\right) \varphi\left(x^{\prime}, 0\right) d x^{\prime} d y & =\lim _{\varepsilon \rightarrow 0} \int_{\Omega} \frac{1}{\varepsilon} \chi_{\varepsilon}(x) v_{\varepsilon}(x) \varphi\left(x^{\prime}, 0\right) d x \\
& =\lim _{\varepsilon \rightarrow 0} \int_{\Omega} \frac{1}{\varepsilon} \chi_{\varepsilon}(x) v_{\varepsilon}(x) \varphi(x) d x \\
& =\int_{\Omega^{\prime}} v\left(x^{\prime}, 0\right) \varphi\left(x^{\prime}, 0\right) d x^{\prime}
\end{aligned}
$$

which implies the desired result, namely that $u\left(x^{\prime}, 0\right)$ is the average on $Y$ of $w\left(x^{\prime}, y\right)$.

The two scale convergence for boundary layers was introduced for time independent problems. It naturally extends to time dependent problems.

Proposition 7. (i) Suppose that $a=-1$. There exists a function $u_{-1}\left(t, x^{\prime}\right) \in$ $L^{2}\left((0, T) ; H^{1}\left(\Omega^{\prime}\right)\right)$ and a function $w\left(t, x^{\prime}, y\right) \in L^{2}\left((0, T) \times \Omega^{\prime} ; H_{l o c, \#}^{1}(G)\right)$ such that, up to a subsequence,

$$
\begin{array}{ccl}
\chi_{\varepsilon} u_{\varepsilon} & \stackrel{2 \mathrm{SBL}}{\longrightarrow} \chi\left(y_{d}\right) u_{-1}\left(t, x^{\prime}\right) \\
\chi_{\varepsilon} \nabla u_{\varepsilon} & \stackrel{2 \mathrm{SBL}}{\longrightarrow} & \chi\left(y_{d}\right)\left(\nabla_{x^{\prime}} u_{-1}\left(t, x^{\prime}\right)+\nabla_{y} w\left(t, x^{\prime}, y\right)\right) .
\end{array}
$$

(ii) Suppose that $a=2$. There exists a function $u_{2}(t, x, y) \in L^{2}\left((0, T) \times \Omega^{\prime} ; H_{l o c, \#}^{1}(G)\right)$ such that, up to a subsequence,

$$
\begin{array}{rll}
\varepsilon u_{\varepsilon} & \stackrel{2 \mathrm{SBL}}{\longrightarrow} u_{2}\left(t, x^{\prime}, y\right) \\
\varepsilon^{2} \nabla u_{\varepsilon} & \stackrel{2 \mathrm{SBL}}{\longrightarrow} \chi\left(y_{d}\right) \nabla_{y} u_{2}\left(t, x^{\prime}, y\right)
\end{array}
$$

Furthermore,

$$
\left(1-\chi\left(y_{d}\right)\right) u_{2}\left(t, x^{\prime}, y\right)=0 \text { for a.e. } y \in G \text {. }
$$


Note. We use the notation

$$
\nabla_{x^{\prime}}=\left(\frac{\partial}{\partial x_{1}}, \ldots, \frac{\partial}{\partial x_{d-1}}, 0\right) .
$$

Similarly, for a $d$ dimensional vector $\Psi$ we shall write

$$
\Psi^{\prime}=\left(\Psi_{1}, \ldots, \Psi_{d-1}, 0\right) .
$$

Proof. Suppose $a=2$. The bound (18) shows that $w_{\varepsilon}=\varepsilon u_{\varepsilon}$ satisfies

$$
\left\|w_{\varepsilon}\right\|_{L^{2}\left((0, T) ; L^{2}(\Omega)\right)}+\varepsilon\left\|\nabla w_{\varepsilon}\right\|_{L^{2}\left((0, T) ; L^{2}(\Omega)^{d}\right)} \leq C \sqrt{\varepsilon} .
$$

Consequently, $w_{\varepsilon}$ (resp. $\varepsilon \nabla w_{\varepsilon}$ ) admits a limit $u_{2}$ (resp. $\zeta_{2}$ ) in the sense of boundary layer two scale limits. It is a classical result [2], [3] that $\varepsilon \nabla w_{\varepsilon}$ two scale converges in the sense of boundary layers to $\zeta_{2}=\nabla_{y} u_{2}$. We shall now prove (31). The bounds (18) show that the sequence $w_{\varepsilon}$ satisfies

$$
\left\|\left(1-\chi_{\varepsilon}\right) w_{\varepsilon}\right\|_{L^{2}\left(0, T ; L^{2}(\Omega)\right)}+\left\|\left(1-\chi_{\varepsilon}\right) \nabla w_{\varepsilon}\right\|_{L^{2}\left(0, T ; L^{2}(\Omega)^{d}\right)} \leq C \sqrt{\varepsilon} .
$$

Therefore, up to a subsequence, $\left(1-\chi_{\varepsilon}\right) \nabla w_{\varepsilon}$ converges in the sense of boundary layers to $\xi_{2}\left(t, x^{\prime}, y\right)$. Since $\chi_{\varepsilon}$ converges strongly in the sense of two-scale boundary layers convergence to $\chi$, we have $\xi_{2}\left(t, x^{\prime}, y\right)=\left(1-\chi\left(y_{d}\right)\right) \xi_{2}\left(t, x^{\prime}, y\right)$. Let $\Psi\left(x^{\prime}, y\right) \in C_{c}^{\infty}\left(\Omega^{\prime} ; C_{\#, c}^{\infty}(G)\right)^{d}$ be a smooth test function such that $\Psi\left(x^{\prime}, y\right)(1-$ $\left.\chi\left(y_{d}\right)\right)=\Psi\left(x^{\prime}, y\right)$, and $\varphi(t) \in C[0, T]$. By an integration by parts we see that

$$
\begin{aligned}
& \lim _{\varepsilon \rightarrow 0} \frac{1}{\varepsilon} \int_{0}^{T} \varphi(t) \int_{\Omega}\left(1-\chi_{\varepsilon}\right) \nabla w_{\varepsilon} \cdot \Psi\left(x^{\prime}, \frac{x}{\varepsilon}\right) d t d x \\
= & -\lim _{\varepsilon \rightarrow 0} \frac{1}{\varepsilon^{2}} \int_{0}^{T} \varphi(t) \int_{\Omega} w_{\varepsilon} \operatorname{div}_{y} \Psi\left(x^{\prime}, \frac{x}{\varepsilon}\right) d t d x^{\prime} d y \\
& -\lim _{\varepsilon \rightarrow 0} \frac{1}{\varepsilon} \int_{0}^{T} \varphi(t) \int_{\Omega} w_{\varepsilon} \operatorname{div}_{x^{\prime}} \Psi\left(x^{\prime}, \frac{x}{\varepsilon}\right) d t d x^{\prime} d y .
\end{aligned}
$$

This implies that

$$
\lim _{\varepsilon \rightarrow 0} \frac{1}{\varepsilon} \int_{0}^{T} \varphi(t) \int_{\Omega} w_{\varepsilon} \operatorname{div}_{y} \Psi\left(x^{\prime}, \frac{x}{\varepsilon}\right) d t d x=0,
$$

that is, $(1-\chi) u_{2}\left(t, x^{\prime}, y\right)$ does not depend on $y$ and is therefore 0 , since $y \mapsto$ $u_{2}(\cdot, \cdot, y) \in L_{\#}^{2}(G)$.

Let us now turn to the case $a=-1$. The bound (17) shows that $\chi_{\varepsilon} u_{\varepsilon}$ and $\chi_{\varepsilon} \nabla u_{\varepsilon}$ admit two scale limits in the sense of boundary layers convergence. Denoting by $w_{-1}$ and $\zeta_{-1}$ these limits, we have for all $\psi\left(x^{\prime}, y\right) \in C_{c}^{\infty}\left(\Omega^{\prime} ; C_{c \#}^{\infty}(G)\right), \Psi\left(x^{\prime}, y\right) \in$ $C_{c}^{\infty}\left(\Omega^{\prime} ; C_{c \#}^{\infty}(G)\right)^{d}$ and $\varphi(t) \in C[0, T]$,

$$
\begin{aligned}
\lim _{\varepsilon \rightarrow 0} \frac{1}{\varepsilon} \int_{0}^{T} \varphi(t) d t \int_{\Omega} \chi_{\varepsilon} u_{\varepsilon} \psi\left(x^{\prime}, \frac{x}{\varepsilon}\right) d x & =\int_{0}^{T} \varphi(t) d t \int_{\Omega^{\prime} \times G} w_{-1}\left(t, x^{\prime}, y\right) \psi\left(x^{\prime}, y\right) d x^{\prime} d y, \\
\lim _{\varepsilon \rightarrow 0} \frac{1}{\varepsilon} \int_{0}^{T} \varphi(t) d t \int_{\Omega} \chi_{\varepsilon} \nabla u_{\varepsilon} \cdot \Psi\left(x^{\prime}, \frac{x}{\varepsilon}\right) d x & =\int_{0}^{T} \varphi(t) d t \int_{\Omega^{\prime} \times G} \zeta_{-1}\left(t, x^{\prime}, y\right) \cdot \Psi\left(x^{\prime}, y\right) d x^{\prime} d y .
\end{aligned}
$$


TWO ASYMPTOTIC MODELS FOR ARRAYS OF UNDERGROUND WASTE CONTAINERS11

We select a test function $\Psi=\left(\Psi_{1}, \ldots, \Psi_{d}\right)$ such that $\Psi\left(x^{\prime}, y\right) \chi\left(y_{d}\right)=\Psi\left(x^{\prime}, y\right)$, integrating by parts and passing to the limit we obtain a condition on $w_{-1}$,

$$
\int_{0}^{T} \varphi(t) d t \int_{\Omega^{\prime} \times G} w_{-1}\left(t, x^{\prime}, y\right) \operatorname{div}_{y} \Psi\left(x^{\prime}, y\right) d x^{\prime} d y=0,
$$

which implies that the two-scale limit $w_{-1}$ of $\chi_{\varepsilon} u_{\varepsilon}$ is independent of $y$ within the support of $\chi$ :

$$
w_{-1}\left(t, x^{\prime}, y\right)=\chi\left(y_{d}\right) u_{-1}\left(t, x^{\prime}\right) .
$$

Selecting now $\Psi^{\prime}=\left(\Psi_{1}, \ldots, \Psi_{d-1}, 0\right)=\Psi^{\prime}\left(x^{\prime}, y\right) \chi\left(y_{d}\right)$ satisfying additionally that $\operatorname{div}_{y} \Psi^{\prime}=0$, we have

$$
\begin{aligned}
& \int_{0}^{T} \varphi(t) d t \int_{\Omega^{\prime} \times G} \xi_{-1}\left(t, x^{\prime}, y\right) \cdot \Psi^{\prime}\left(x^{\prime}, y\right) d x^{\prime} d y \\
= & -\int_{0}^{T} \varphi(t) d t \int_{\Omega^{\prime} \times G} \chi\left(y_{d}\right) u_{-1}\left(t, x^{\prime}\right) \operatorname{div}_{x^{\prime}} \Psi^{\prime}\left(x^{\prime}, y\right) d x^{\prime} d y \\
= & \int_{0}^{T} \varphi(t) d t \int_{\Omega^{\prime} \times G} \chi\left(y_{d}\right) \nabla_{x^{\prime}} u_{-1}\left(t, x^{\prime}\right) \cdot \Psi^{\prime}\left(x^{\prime}, y\right) d x^{\prime} d y .
\end{aligned}
$$

Since the orthogonal set in $L^{2}\left(\Omega^{\prime} \times Y\right)$ of all $Y^{\prime}$-periodic functions $\Psi^{\prime}$ satisfying the constraint $\operatorname{div}_{y} \Psi^{\prime}=0$ is precisely the set of all gradients $\nabla_{y} w\left(x^{\prime}, y\right)$ with $w \in L^{2}\left(\Omega^{\prime} ; H_{\#}^{1}(Y)\right)$ (recall that these last functions are not periodic in the last $y_{d}$ variable), we have proved that

$$
\zeta_{-1}\left(t, x^{\prime}, y\right)=\chi\left(y_{d}\right) \nabla_{x^{\prime}} u_{-1}\left(t, x^{\prime}\right)+\chi\left(y_{d}\right) \nabla_{y} w\left(t, x^{\prime}, y\right) .
$$

\section{Proof of Theorem 1}

For any test function $\psi\left(x^{\prime}, x / \varepsilon\right)$ such that $\psi\left(x^{\prime}, y\right) \chi\left(y_{d}\right)=\psi\left(x^{\prime}, y\right)$, and any smooth function $\varphi(t)$ such that $\varphi(0)=\varphi(T)=0$, we obtain from Proposition 7 that

$$
\begin{aligned}
\varepsilon \int_{0}^{T} \varphi(t) d t \int_{\Omega} \rho_{\varepsilon}(x) \frac{\partial u_{\varepsilon}}{\partial t} \psi\left(x^{\prime}, x / \varepsilon\right) d x & =-\int_{0}^{T} d t \int_{\Omega^{\prime}} \int_{G} \psi\left(x^{\prime}, y\right) \rho_{1}(y) u_{2}\left(t, x^{\prime}, y\right) \frac{\partial \varphi}{\partial t} d x^{\prime} d y+o(1), \\
\int_{0}^{T} \varphi(t) d t \int_{\Omega} \varepsilon^{2} A_{\varepsilon} \nabla u_{\varepsilon} \cdot \nabla \psi\left(x^{\prime}, x / \varepsilon\right) d x & =\int_{0}^{T} \varphi d t \int_{\Omega^{\prime}} \int_{G} \mu_{1}(y) \nabla_{y} u_{2}\left(t, x^{\prime}, y\right) \cdot \nabla_{y} \psi\left(x^{\prime}, y\right) d x^{\prime} d y+o(1), \\
\varepsilon \int_{0}^{T} \varphi(t) d t \int_{\Omega} \omega_{\varepsilon} u_{\varepsilon} \psi\left(x^{\prime}, x / \varepsilon\right) d x & =\int_{0}^{T} \varphi(t) d t \int_{\Omega^{\prime}} \int_{G} \omega(y) u_{2}\left(t, x^{\prime}, y\right) \psi\left(x^{\prime}, y\right) d x^{\prime} d y+o(1), \\
\int_{0}^{T} \varphi(t) d t \int_{\Omega} f_{\varepsilon}(x) \psi\left(x^{\prime}, x / \varepsilon\right) d x & =\int_{0}^{T} \varphi(t) d t \int_{\Omega^{\prime}} \int_{G} f_{1}(t, y) \psi\left(x^{\prime}, y\right) d x^{\prime} d y+o(1) .
\end{aligned}
$$

Here, as in Proposition $7, u_{2}$ is the limit of $\varepsilon u_{\varepsilon}$. Combining these terms as in (1) we deduce that $u_{2}$ satisfies the limit system

$$
\begin{aligned}
\rho_{1}(y) \frac{\partial u_{2}}{\partial t}-\operatorname{div}_{y}\left(\mu_{1}(y) \nabla_{y} u_{2}\right)+\omega_{1}(y) u_{2} & =f_{1}(t, y) \text { in } \Omega^{\prime} \times Y, \text { for a.e. } t \\
u_{2}\left(t, x^{\prime}, y\right) & =0 \text { on } \Omega^{\prime} \times Y^{\prime} \times\left\{\left|y_{d}\right|=1 / 2\right\}, \text { for a.e. } t \\
u_{2}(t=0) & =0 .
\end{aligned}
$$

Note that $u_{2}$ is independent of $x^{\prime}$ : thus, system (32) is in fact (8), and our notations are consistent. In the sequel we shall write $u_{2}(t, y)$, the unique solution in 
$C\left([0, T] ; H_{\#}^{1}(Y)\right)$ of (8). This first result, the characterization of the limit behavior of $\varepsilon u_{\varepsilon}$, does not provide a detailed description of the asymptotic behavior of the solution of (1), since the support of $u_{2}(\cdot, \dot{\bar{\varepsilon}})$ shrinks to zero with $\varepsilon$. Consequently, we turn to the second order term, and define

$$
v_{\varepsilon}(t, x)=u_{\varepsilon}(t, x)-\frac{1}{\varepsilon} u_{2}\left(t, \frac{x}{\varepsilon}\right) \text { for a.e. }(t, x) \in[0, T] \times \Omega .
$$

The following gives the equation satisfied by $v_{\varepsilon}$.

Proposition 8. The solution $u_{\varepsilon}$ of problem (1) can be written

$$
u_{\varepsilon}(t, x)=\frac{1}{\varepsilon} u_{2}(t, x / \varepsilon)+v_{\varepsilon}(t, x), \text { for a.e. }(t, x) \in[0, T] \times \Omega,
$$

where $u_{2}$ is the unique solution of system (32). The remainder term $v_{\varepsilon}$ satisfies

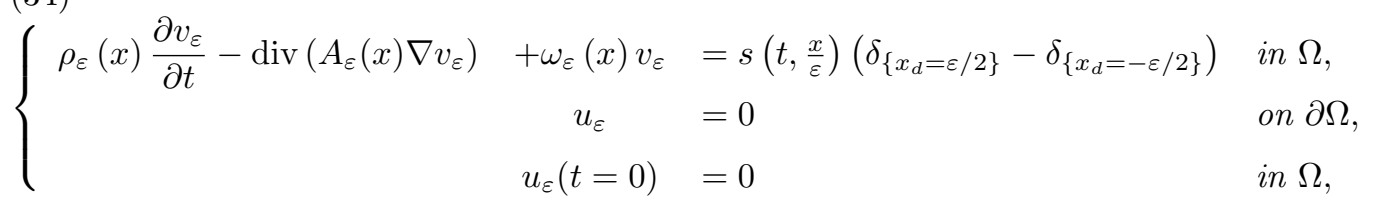

where

$$
s(t, y)=\mu_{1}(y) \frac{\partial u_{2}}{\partial y_{d}}(t, y) .
$$

Furthermore, $v_{\varepsilon}$ satisfies the following a priori estimate

$\left\|v_{\varepsilon}\right\|_{L^{\infty}\left((0, T) ; L^{2}(\Omega)\right)}+\left\|\left(1-\chi_{\varepsilon}\right) \nabla v_{\varepsilon}\right\|_{L^{2}\left((0, T) ; L^{2}(\Omega)^{d}\right)}+\varepsilon\left\|\chi_{\varepsilon} \nabla v_{\varepsilon}\right\|_{L^{2}\left((0, T) ; L^{2}(\Omega)^{d}\right)} \leq C$,

where $C$ is a constant independent of $\varepsilon$.

Proof. System (34) is obtained without difficulty from (1) and (32). From assumption (5) we deduce that there exists $\eta>0$ such that

$$
u_{2} \in L^{\infty}\left((0, T) ; C^{1, \eta}\left(Y^{\prime} \times[-1 / 2,-1 / 2+\eta] \cup[1 / 2-\eta, 1 / 2]\right)\right)
$$

(see e.g. [11, Thm 8.34]). As a consequence, $s(t, x / \varepsilon)$ is continuous, and bounded around $x_{d}= \pm \varepsilon / 2$. Since in the right hand side of (34) $s$ is multiplied by Dirac functions located at $x_{d}= \pm \varepsilon / 2$, we can assume without loss of generality that $s(t, x / \varepsilon) \in C([0, T] \times \Omega)$. Then, estimate (36) is a consequence of $(15)$.

The conclusion of the proof of Theorem 1 is given by the following proposition.

Proposition 9. The solution $v_{\varepsilon}$ of (34) is such that $\left(1-\chi_{\varepsilon}\right) v_{\varepsilon}$ converges strongly in $L^{2}\left((0, T) \times \Omega^{ \pm}\right)$to $u^{ \pm}$and $\left(1-\chi_{\varepsilon}\right) \nabla v_{\varepsilon}$ converges weakly in $L^{2}\left((0, T) \times \Omega^{ \pm}\right)^{d}$ to $\nabla u^{ \pm}$, where $u^{ \pm}$is the solution of the homogenized problem (6).

Proof. We focus on the convergence to $u^{+}$, the convergence to $u^{-}$can be proved by similar arguments. The bound (36) on $\left\|v_{\varepsilon}\right\|_{L^{\infty}\left(0, T, L^{2}(\Omega)\right)}$ shows that, up to the possible extraction of a subsequence, there exists $v_{0}(t, x) \in L^{2}\left((0, T) ; L^{2}\left(\Omega^{+}\right)\right)$such that for $v_{\varepsilon}$ converges weakly in $L^{2}\left((0, T) ; L^{2}(\Omega)\right)$ to $v_{0}$. Similarly, the bound (36) 
TWO ASYMPTOTIC MODELS FOR ARRAYS OF UNDERGROUND WASTE CONTAINERS13

on $\left\|\left(1-\chi_{\varepsilon}\right) \nabla v_{\varepsilon}\right\|_{L^{2}\left((0, T) ; L^{2}(\Omega)^{d}\right)}$ shows that, up to the possible extraction of a subsequence, there exists $\xi_{0}(t, x) \in L^{2}\left((0, T) ; L_{2}(\Omega)^{d}\right)$ such that for $\left(1-\chi_{\varepsilon}\right) \nabla v_{\varepsilon}$ converges weakly in $L^{2}\left((0, T) ; L^{2}(\Omega)^{d}\right)$ to $\xi_{0}$. For any $\Phi \in C_{c}^{1}\left((0, T) \times\left(\Omega^{+} \cup \Omega^{-}\right)\right)^{d}$, one easily obtains that

$$
\int_{0}^{T} \int_{\Omega^{+} \cup \Omega^{-}} \xi_{0} \cdot \Phi d t d x=-\int_{0}^{T} \int_{\Omega^{+} \cup \Omega^{-}} v_{0} \operatorname{div} \Phi d t d x
$$

or in other words that $\xi_{0}=\nabla v_{0}$ on $\Omega^{+} \cup \Omega^{-}$. Let $\theta \in C^{2}(\mathbb{R})$ be a non-negative one dimensional cut-off function, such that $\theta(s)=1$ for $s \geq 1 / 2$ and $\theta(s)=0$ for $s \leq-1 / 2$, and define

$$
\theta_{\varepsilon}(x)=\theta\left(\frac{x_{d}}{\varepsilon}\right) \quad x \in \mathbb{R}^{d} .
$$

Given a test function $\varphi \in C^{1}\left((0, T) ; H_{0}^{1}(\Omega)\right)$, such that $\varphi(T, \cdot)=0$, we test system (34) against $\varphi \theta_{\varepsilon}$ to obtain

$$
\begin{aligned}
& \int_{0}^{T} d t \int_{\Omega^{+}} d x\left(1-\chi_{\varepsilon}\right) \theta_{\varepsilon}(x) v_{\varepsilon}(x)\left(-\rho_{0}(x) \frac{\partial \varphi}{\partial t}+\omega_{0}(x) \varphi\right) \\
+ & \int_{0}^{T} d t \int_{\Omega^{+}} d x\left(1-\chi_{\varepsilon}\right) \mu_{0}(x) \nabla v_{\varepsilon}(x) \cdot \nabla \varphi \\
+ & \int_{0}^{T} d t \int_{\Omega^{\prime}} d x^{\prime} s\left(t, \frac{x^{\prime}}{\varepsilon}, 1 / 2\right) \varphi\left(t, x^{\prime}, \varepsilon\right) \\
= & \int_{0}^{T} d t \int_{\Omega} d x \chi_{\varepsilon} \theta_{\varepsilon} v_{\varepsilon}\left(\rho_{\varepsilon}(x) \frac{\partial \varphi}{\partial t}-\omega_{\varepsilon}(x) \varphi\right) \\
- & \int_{0}^{T} d t \int_{\Omega} d x \varepsilon^{2} \chi_{\varepsilon} \mu_{1}\left(\frac{x}{\varepsilon}\right) \nabla v_{\varepsilon} \cdot \nabla\left(\theta_{\varepsilon} \varphi\right) .
\end{aligned}
$$

Thanks to (36), the first right-hand side term is bounded by

$$
\sqrt{\varepsilon}\left\|\left(\rho_{1} \frac{\partial \varphi}{\partial t}-\omega_{1} \varphi\right)\right\|_{L^{\infty}\left((0, T) ; L^{2}(\Omega)\right)}\left\|v_{\varepsilon}\right\|_{L^{\infty}\left((0, T) ; L^{2}(\Omega)\right)} \leq C \sqrt{\varepsilon},
$$

whereas the second right-hand side term is bounded by

$\varepsilon\left\|\chi_{\varepsilon} \nabla v_{\varepsilon}\right\|_{L^{2}\left((0, T) ; L^{2}(\Omega)^{d}\right)}\left\|\mu_{1}\right\|_{L^{\infty}\left(\mathbb{R}^{d}\right)}\left(\varepsilon\|\nabla \varphi\|_{L^{\infty}\left((0, T) ; L^{2}(\Omega)^{d}\right)}+\sqrt{\varepsilon}\|\varphi\|_{L^{\infty}\left((0, T) ; L^{2}(\Omega)\right)}\|\chi \nabla \theta\|_{L^{2}\left(\mathbb{R}^{d}\right)}\right) \leq C \sqrt{\varepsilon}$.

Passing to the limit in the left-hand side, we obtain

$\int_{0}^{T} \int_{\Omega^{+}} v_{0}\left(-\rho_{0}(x) \frac{\partial \varphi}{\partial t}+\omega_{0}(x) \varphi\right)+\int_{0}^{T} \int_{\Omega^{+}} \mu_{0}(x) \nabla v_{0} \cdot \nabla \varphi-\int_{0}^{T} \int_{\Omega^{\prime}} s^{+}(t) \varphi\left(t, x^{\prime}, 0\right) d x^{\prime}=0$.

which is the system (6) satisfied by $u^{+}$. The limit system for $u^{-}$is obtained similarly.

\section{Proof of Theorem 2}

Thanks to the a priori estimate (16) we know that $u_{\varepsilon}$ is a bounded sequence in $L^{\infty}\left((0, T) ; H_{0}^{1}(\Omega)\right)$. It therefore admits a weak limit $u$, up to the extraction of a subsequence, in $L^{2}\left((0, T) ; H_{0}^{1}(\Omega)\right)$. We can now pass to the limit in (1) in any 
subdomain $\omega \subset \Omega$ not intersecting the plane $x_{d}=0$. Furthermore, thanks to Lemma 6 , we know that for a.e. $x^{\prime} \in \Omega^{\prime}$ and every $t>0$,

$$
u\left(t, x^{\prime}, 0\right)=\int_{G} \chi\left(y_{d}\right) u_{-1}\left(t, x^{\prime}\right) d y=u_{-1}\left(t, x^{\prime}\right) .
$$

We therefore have proved that, up to a subsequence, $u$ satisfies

$$
\left\{\begin{aligned}
\rho_{0}(x) \frac{\partial u}{\partial t}-\operatorname{div}\left(\mu_{0}(x) \nabla u\right)+\omega_{0}(x) u & =0 & & \text { in } \Omega \\
u\left(t, x^{\prime}, 0\right) & =u_{-1}\left(t, x^{\prime}\right) & & \text { on } \Omega^{\prime} \\
u(t=0) & =0 & & \text { in } \Omega, \\
u & =0 & & \text { on } \partial \Omega .
\end{aligned}\right.
$$

Let us now turn to the derivation of equation (10) to conclude the proof.

The following proposition determines $w(t, x, y)$, defined in Proposition 7 , in terms of $u_{-1}\left(t, x^{\prime}\right)$.

Proposition 10. The two-scale limit of $\chi_{\varepsilon} \nabla u_{\varepsilon}$ is precisely $\chi\left(y_{d}\right)\left(I_{d}+P(y)\right) \nabla_{x^{\prime}} u_{-1}\left(t, x^{\prime}\right)$ where $I_{d}$ is the identity matrix of $\mathbb{R}^{d}$, and $P(y)$ is the matrix-valued function defined by $P(\cdot) e_{i}=\nabla \varphi_{i}$, for $i=1, \ldots, d$, where $\varphi_{i} \in H_{\#, d}^{1}(Y)$ is the solution of (13).

Proof. For any $\psi\left(x^{\prime}, y\right) \in C\left(\Omega^{\prime} ; L_{\#}^{2}(G)\right)$ and $\varphi(t) \in C_{c}^{1}(0, T)$, testing (1) against $\varepsilon \varphi(t) \psi\left(x^{\prime}, x / \varepsilon\right)$ yields

$$
\int_{0}^{T} \varphi(t) d t \int_{\Omega} \chi_{\varepsilon}(x) \mu_{1}\left(\frac{x}{\varepsilon}\right) \nabla u_{\varepsilon} \cdot\left(\nabla_{x^{\prime}} \psi+\frac{1}{\varepsilon} \nabla_{y} \psi\right)=o(1) .
$$

Since $\mu_{1}(y)$ is periodic, it converges strongly with respect to two-scale convergence and we have

$$
\begin{aligned}
& \frac{1}{\varepsilon} \int_{0}^{T} \varphi(t) d t \int_{\Omega} \mu_{1}\left(\frac{x}{\varepsilon}\right) \chi_{\varepsilon}(x) \nabla u_{\varepsilon} \cdot \nabla_{y} \psi d x \\
& =\int_{0}^{T} \varphi(t) d t \int_{\Omega^{\prime} \times G} \mu_{1}(y) \chi\left(y_{d}\right)\left(\nabla_{x^{\prime}} u_{-1}\left(t, x^{\prime}\right)+\nabla_{y} w\left(t, x^{\prime}, y\right)\right) \cdot \nabla_{y} \psi d x^{\prime} d y+o(1) .
\end{aligned}
$$

The above limit is just the variational formulation, for a.e. $x \in \Omega^{\prime}$ and $t>0$, of

$$
\operatorname{div}_{y}\left(\mu_{1}(y)\left(\nabla_{x^{\prime}} u_{-1}\left(t, x^{\prime}\right)+\nabla_{y} w\left(t, x^{\prime}, y\right)\right)\right)=0 \text { in } y \in Y
$$

with Neumann boundary conditions on the lower and upper faces as in (13).

Let us now test $(1)$ against $\varphi \in C_{c}^{1}((0, T) \times \Omega)$ with $\varphi(T, \cdot)=0$. We obtain

$$
\begin{aligned}
\int_{0}^{T} d t \int_{\Omega} d x\left(1-\chi_{\varepsilon}\right)\left(u_{\varepsilon}\left(-\rho_{0}(x) \frac{\partial \varphi}{\partial t}+\omega_{0}(x) \varphi\right)+\mu_{0}(x) \nabla u_{\varepsilon} \cdot \nabla \varphi\right) \\
+\int_{0}^{T} d t \int_{\Omega} d x \chi_{\varepsilon}\left(u_{\varepsilon}\left(-\rho_{1}\left(\frac{x}{\varepsilon}\right) \frac{\partial \varphi}{\partial t}+\omega_{1}\left(\frac{x}{\varepsilon}\right) \varphi\right)+\frac{1}{\varepsilon} \mu_{1}\left(\frac{x}{\varepsilon}\right) \nabla u_{\varepsilon} \cdot \nabla \varphi\right) \\
-\int_{0}^{T} d t \int_{\Omega} \chi_{\varepsilon} \frac{1}{\varepsilon} \varphi f_{1} d x=0 .
\end{aligned}
$$


TWO ASYMPTOTIC MODELS FOR ARRAYS OF UNDERGROUND WASTE CONTAINERS15

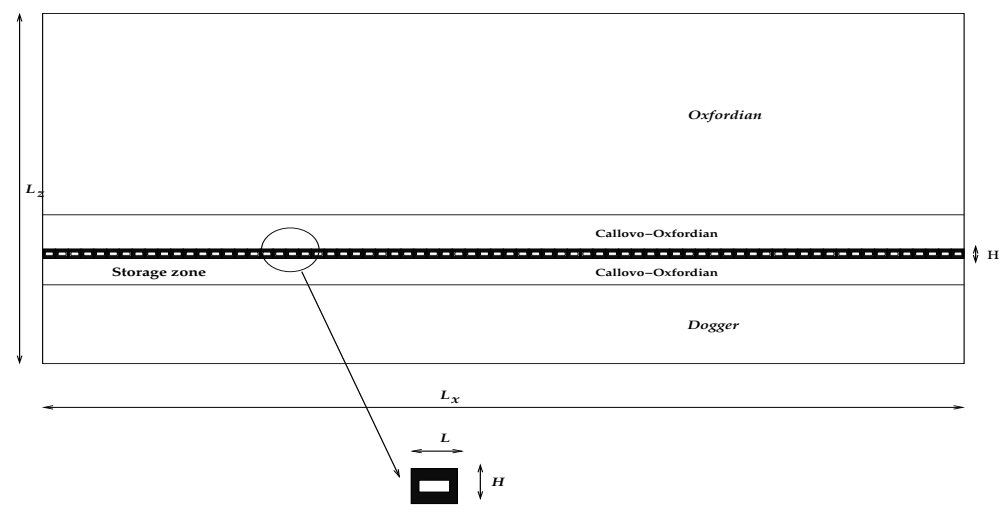

FiguRE 2. Nuclear waste repository and surrounding geological layers.

In order to pass to the limit as $\varepsilon$ tends to zero, remark that $\chi_{\varepsilon} \varphi$ or $\chi_{\varepsilon} \nabla \varphi$ are admissible test functions for two-scale convergence in the sense of boundary layers.

As a consequence, we obtain

$$
\begin{aligned}
\int_{0}^{T} d t \int_{\Omega} d x & \left(u\left(-\rho_{0}(x) \frac{\partial \varphi}{\partial t}\left(t, x^{\prime}, x_{d}\right)+\omega_{0}(x) \varphi\left(t, x^{\prime}, x_{d}\right)\right)+\mu_{0}(x) \nabla u \cdot \nabla \varphi\left(t, x^{\prime}, x_{d}\right)\right) \\
+\int_{0}^{T} d t \int_{\Omega^{\prime}} \int_{G} \chi\left(y_{d}\right)\left(\mu_{1}(y)\left(I_{d}+P(y)\right)\right) \nabla_{x^{\prime}} u_{-1}\left(t, x^{\prime}\right) \cdot \nabla_{x^{\prime}} \varphi\left(t, x^{\prime}, x_{d}\right) d y d x^{\prime} & \\
-\int_{0}^{T} d t \int_{\Omega^{\prime}} d x^{\prime} \int_{G} \chi\left(y_{d}\right) f_{1}(t, y) \varphi\left(t, x^{\prime}, x_{d}\right) d y & =0 .
\end{aligned}
$$

This last system is the weak formulation of (9)-(10),

$\left.\int_{0}^{T} \int_{\Omega} F(u, \varphi) d t d x+\int_{0}^{T} \int_{\Omega^{\prime}} F^{\prime}\left(u\left(t, x^{\prime}, 0\right), \varphi\left(t, x^{\prime}, 0\right)\right) d t d x^{\prime}=\int_{0}^{T} \int_{\Omega^{\prime}} \bar{f}(t) \varphi\left(t, x^{\prime}, 0\right)\right) d t d x^{\prime}$,

where $\bar{f}(\mathrm{t})$ is given by (11), $F$ and $F^{\prime}$ are the bilinear forms

$$
\begin{gathered}
F(u, v)=u\left(-\rho_{0} \frac{d v}{d t}+\omega_{0} v\right)+\mu_{0} \nabla_{x} u \cdot \nabla_{x} v, \\
F^{\prime}(u, v)=\mu^{*} \nabla_{x^{\prime}} u \cdot \nabla_{x^{\prime}} v,
\end{gathered}
$$

with $\mu^{*}$ being the $(d-1)$-dimensional periodic homogenized diffusion coefficient given by (12).

\section{Numerical simulation of a nUClear Waste storage}

In this section, we revisit the scaling of our model (1). Introducing appropriate scales, we perform an adimensionalization and introduce the small parameter $\varepsilon$. Then, using some experimental values of the diffusion coefficients, we show that the dimensionless derived model is of the same type as (1). Relying on the previously obtained homogenized problems we perform numerical simulations of the long time behavior of the nuclear waste storage. These computations are done with the FreeFem ++ software which may be downloaded from [10]. 
6.1. Nuclear waste repository. The storage of nuclear waste is achieved within glass containers in groups of 10, inside alveolus situated on both sides of 3 galleries. This set is called module, following the ANDRA (French National Radioactive Waste Management Agency) terminology.

A large number of disposal modules makes up a storage zone. The whole repository system lies at a depth of about four to five hundred meters in the 130 meters thick geological argillite layer called Callovo-Oxfordian formation. Above this layer, the Oxfordian formation (400 meters height) is considered as the first geological limestone underground layer. Bellow, the Dogger formation (150 meters height) is a limestone layer. The computational domain is made of these three geological layers. This zone is on the whole $L_{z}=684$ meters deep (see Figure 3).

Modeling all the details of a repository is difficult and it is a common practice to use a simplified model based on the homogenization of the storage zone [1]. In our approach, the cylindrical unit of storage (1.5 meters height and 1 meter diameter) containing the radioactive waste is surrounded by material such as concrete, clay, argillite or bentonite. This is what the simplified elementary cell is composed of. In our 2-d setting the cell has height $H=3$ meters and length $L=3$ meters. A large number of these elementary cells, 3 meters distant from each other, lie horizontally, overlapping the reference plane $(z=0)$ located in the middle of the Callovo-Oxfordian.

6.2. Scale analysis of the diffusion problem. In first approximation the radionucleide transport is governed by a pure diffusion process [1]. Thus, the radionucleide concentration $C$ satisfies the diffusion equation

$$
R \omega\left(\frac{\partial C}{\partial t}+\mu C\right)-\operatorname{div}\{D \nabla C\}=F \text { in } \mathcal{O}
$$

where $R$ is the latency retardation factor, $\omega$ the effective porosity and $\mu$ is related to the half life time $\tau$ of the element by the relation $\mu=\log 2 / \tau$. We denote by $D$ the effective diffusion tensor. Here, $\mathcal{O}$ is the 2 -d $(O x z)$ plane section of the repository surrounded with its geological layers and we only consider the section given by $L_{x}=\gamma L_{z}$ with $\gamma \geq 1$. All the containers are supposed to be cut by this plane (see Figure 3$)$. The source term $F$ is related to experimental data $\tilde{f}(t)($ moles $/$ year $)$ by the relation $F(t)=\tilde{f}(t) / S$ where $S$ is the surface occupied by the nuclear waste and is zero outside the containers.

In the following, we use the subscript ref for characteristic or reference values of the parameters and variables involved. Setting

$$
x_{a}=\frac{x}{\gamma L_{\mathrm{ref}}}, z_{a}=\frac{z}{L_{\mathrm{ref}}}, D_{a}=\frac{D}{D_{\mathrm{ref}}}, R_{a}=\frac{R}{R_{\mathrm{ref}}}, \omega_{a}=\frac{\omega}{\omega_{\mathrm{ref}}}, t_{a}=\frac{t}{T_{\mathrm{ref}}}
$$

and omitting the subscript "a " for the dimensionless parameters and variables, the equation for the transport of the radioactive elements in each medium takes the general form

$$
\frac{R_{\mathrm{ref}} \omega_{\mathrm{ref}}}{T_{\mathrm{ref}}} R \omega\left(\frac{\partial C}{\partial t}+\tilde{\lambda} C\right)-\operatorname{div}\left\{\frac{D_{\mathrm{ref}}}{L_{\mathrm{ref}}^{2}} D_{\gamma} \nabla C\right\}=F\left(T_{\mathrm{ref}} t\right)
$$



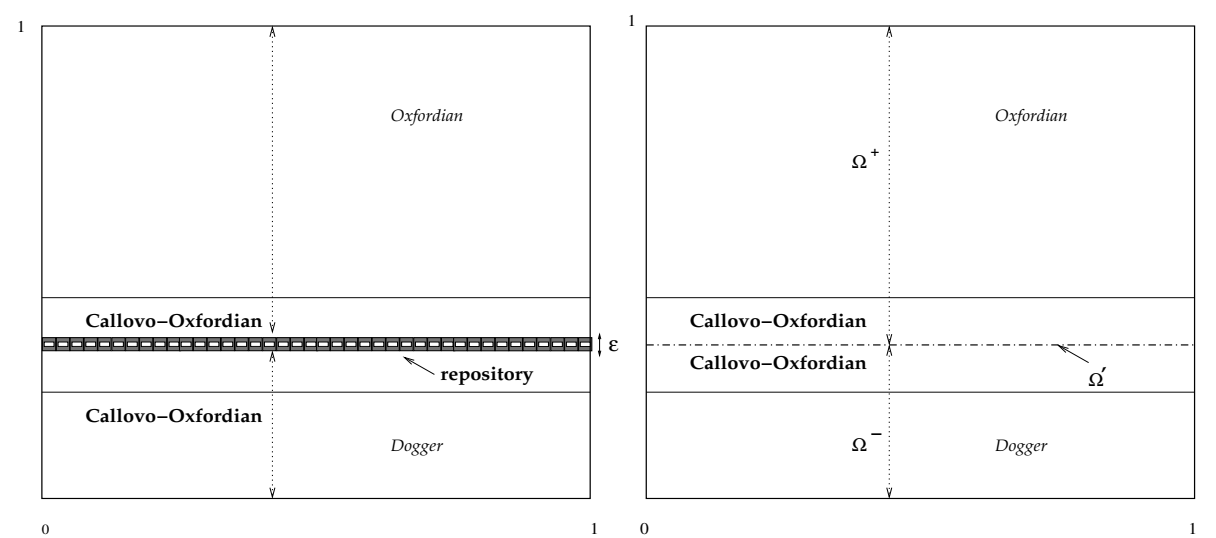

Figure 3. The computational domain.

where

$$
D_{\gamma}=D\left(\begin{array}{rr}
\gamma^{-2} & 0 \\
0 & 1
\end{array}\right)
$$

Here $\tilde{\lambda}$ stands for $\mu T_{\text {ref }}$. The concentration $C$ takes its values in the rescaled domain $\Omega=] 0,1[\times] 0,1\left[\right.$ (see Figure 3 ) composed by the subdomains denoted $\Omega_{\text {sup }}$ (limestone layer), $\Omega_{\mathrm{COX}}$ (argillite layer) and $\Omega_{\text {inf }}$ (limestone layer).

After dividing by $R_{\text {ref }} \omega_{\text {ref }} / T_{\text {ref }}$, equation (37) reads

$$
R \omega\left(\frac{\partial C}{\partial t}+\tilde{\lambda} C\right)-\operatorname{div}\left\{\frac{D_{\text {ref }} T_{\text {ref }}}{L_{\text {ref }}^{2} R_{\text {ref }} \omega_{\text {ref }}} D_{\gamma} \nabla C\right\}=F \frac{T_{\text {ref }}}{R_{\text {ref }} \omega_{\text {ref }}} \text { in } \Omega
$$

The adimensionalization relation

$$
T_{\text {ref }}=\frac{L_{\mathrm{ref}}^{2} \rho_{\mathrm{ref}}}{D_{\mathrm{ref}}}
$$

defines the reference time. The surface $S$ occupied by the $L_{x} / L$ containers represents a percentage $\delta$ of the thin strip in which they are stored

$$
S=\delta \gamma L_{\mathrm{ref}} H
$$

Introducing the concentration reference value

$$
C_{\mathrm{ref}}=\max (\tilde{f}) \frac{1}{\delta \gamma D_{\mathrm{ref}}},
$$

the dimensionless transport equation for the rescaled concentration $U=C / C_{\text {ref }}$ is

$$
R \omega\left(\frac{\partial U}{\partial t}+\tilde{\lambda} U\right)-\operatorname{div}\left\{D_{\gamma} \nabla U\right\}=\frac{L_{\mathrm{ref}}}{H} f \operatorname{in} \Omega
$$

where $f=\tilde{f} / \max (\tilde{f})$ inside the containers and zero outside. 
6.3. Small parametric dependences. We choose the "observation distance" $L_{\text {ref }}=L_{z}$ as the reference distance value and we introduce the small parameter $\varepsilon$ as the ratio of the repository height over the reference length: $\varepsilon=\frac{H}{L_{\mathrm{ref}}}\left(\simeq 4.3810^{-3}\right)$. The $L_{x} / L$ containers are distributed periodically over $\Omega^{\prime}$ with period $\varepsilon$. They are surrounded by a coating (or buffer) material.

Typical values for diffusion coefficients in the case of ${ }^{129} I$ (Iodine 129 ) are $1.8910^{-2} \mathrm{~m}^{2} /$ year for concrete [15] and $9.4810^{-7}$ [8] for the argillite. The choice of concrete diffusion coefficient, as reference value, gives a dimensionless coefficient for surrounding material equal to $5.0110^{-5}$ (which is of the same order of magnitude as $\varepsilon^{2} \simeq 1.910^{-5}$ ) and corresponds to the first case $(a=2)$.

Another typical value for diffusion coefficient of the argillite (always in the case of ${ }^{129} I$ and with the same value for concrete) is $1.5810^{-4} \mathrm{~m}^{2} /$ year [15]. With this value as reference value the dimensionless coefficient for concrete equal to 120 (which is of the same order of magnitude as $\varepsilon^{-1} \simeq 228$ ) and corresponds to the second case $(a=-1)$.

This simple scaling computation is thus a justification of our choice of $\varepsilon$-scaling in (1). In other words, the diffusion tensor $A^{\varepsilon}$ scales like $\varepsilon^{-1}$ or $\varepsilon^{2}$, i.e., takes the form

$$
A^{\varepsilon}(x, z)=\varepsilon^{a} A(x / \varepsilon, z / \varepsilon)\left(\begin{array}{rr}
\gamma^{-2} & 0 \\
& \\
0 & 1
\end{array}\right) \quad \text { with } a=-1 \text { or } 2,
$$

in the thin strip $|x|<\varepsilon / 2$ where

$$
A(x / \varepsilon, z / \varepsilon)=\alpha_{1} \chi_{\mathrm{W}}^{\varepsilon}\left((x, z)+\alpha_{2}\left(1-\chi_{\mathrm{W}}^{\varepsilon}((x, z)) .\right.\right.
$$

In (39), $\alpha_{1}$ denotes the diffusion constant for the vitrified nuclear waste, $\alpha_{2}$ the one of the buffer material and $\chi_{\mathrm{W}}^{\varepsilon}(x, z)=\chi_{\mathrm{W}}\left(\left(\frac{x}{\varepsilon}, \frac{z}{\varepsilon}\right)\right.$ the characteristic function of the waste into the computational domain. For $|x|>\varepsilon / 2$, the physical values of the diffusion constants of the others geological layers define the diffusion tensor $A^{\varepsilon}$ of order $\varepsilon^{0}$.

In the same way, we denote by $\rho^{\varepsilon}$ (resp. $\omega^{\varepsilon}$ ) the bounded function corresponding to the products $R \omega$ (resp. $\rho^{\varepsilon} \tilde{\lambda}$ ) in the different subdomains of $\Omega$. Introducing a right hand side or source term, which is of average of order $\varepsilon^{0}$,

$$
f^{\varepsilon}=\varepsilon^{-1} f \chi_{\mathrm{W}}^{\varepsilon}((x, z),
$$

the adimensionalized diffusion equation reads

$$
\rho^{\varepsilon} \frac{\partial u^{\varepsilon}}{\partial t}-\operatorname{div}\left\{A^{\varepsilon} \nabla u^{\varepsilon}\right\}+\lambda^{\varepsilon} u^{\varepsilon}=f^{\varepsilon} \text { in } \Omega
$$

where the concentration in the different subdomains is denoted $u^{\varepsilon}$. Of course, we assume perfect transmission conditions at the interfaces between geological layers, namely continuity of the concentration and of the normal flux. As a final remark, let us mention that we have ignored convection phenomena which can take place, most notably in the upper and lower geological layers. This is just for simplicity and there is no conceptual difficulty to include them in our model as well. 
6.4. Numerical simulation of a nuclear waste storage. Our asymptotic analysis in the previous sections leads to two different homogenized problems according to the value of the exponent $a=-1,2$. The diffusion coefficients for the storage zone are constant, equal to $\varepsilon^{a}$ with $a=-1,2$. All other physical constants are taken to be equal to one. We make a comparison between direct simulations of the original model (1) and reconstructed solutions of the homogenized models. The simulations concern the direct problems and its asymptotic ones. The source term is a hat function which takes its maximum value $(f=1)$ in the middle of the time interval $(0,1)$ :

$$
f(t)= \begin{cases}2 t & \text { for } 0 \leq t \leq 1 / 2 \\ 2-2 t & \text { for } 1 / 2 \leq t \leq 1 \\ 0 & \text { for } t \geq 1\end{cases}
$$

All computations are perform on a unit square domain $\Omega(\gamma=1)$. The numerical approximation of such parabolic boundary value problems is classical: we use a $P_{1}$ finite element method to discretize the variational formulation in space and an Euler implicit scheme for the time integration in the FreeFem ++ software [10]. Time integration is perform until $t=1$ in 120 time-steps. For the two direct problems $(a=-1,2)$, the same triangular mesh is used ( $\simeq 34000$ degrees of freedom). The corresponding Callovo-Oxfordian zone, surrounding the waste, is meshed more finely than the rest of the computational domain.

6.4.1. The case of small diffusion coefficient: $a=2$. In this case, the asymptotic model (6) has a time-dependent Neumann type boundary condition on the asymptotic limit surface occupied by the storage zone. This emitting surface is the support of effective boundary conditions which supply a source term for the partial differential equation describing the diffusion of the concentration of radionucleides into geological medium surrounding the repository. The numerical simulation of the direct problem (1) shows a decoupling (see Figure 4(a)) of the solution at the emitting surface between the upper and lower parts of the computational domain $\Omega$.

The solution of the direct problem (1) remains highly concentrated in the storage zone (see Figure 4(b)) which weakly emits towards the rest of the domain.

We compare this solution with that of the asymptotic problem (6) where the source terms $s^{+}$and $s^{-}$, defined by (7), are computed via the numerical integration of the parabolic problem (8) associated to $u_{2}$. In the parabolic boundary value problem (8), all coefficients are constant, equal to one. Eventually, $u_{2}$ is computed on a very fine mesh (see Figure 5) of the elementary cell (a unit square containing the nuclear waste) with a classical $P_{2}$ finite element method and $s^{+}$(resp. $s^{-}$) by a standard quadrature formula.

Then, using two different triangular meshes for, respectively, the upper and lower parts of the computational domain (with respectively $\simeq 7600$ and $\simeq 3400$ degrees of freedom), the computation of the solution of the asymptotic problem (see Figure 6) is achieved.

The decoupling phenomenon, appearing in the asymptotic problem formulation, can be illustrated by Figure 7 which allows to compare the truncated solution of the direct problem in the storage zone and the one of the asymptotic problem. 


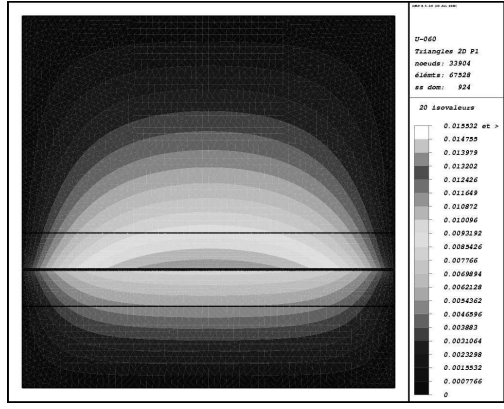

(a)

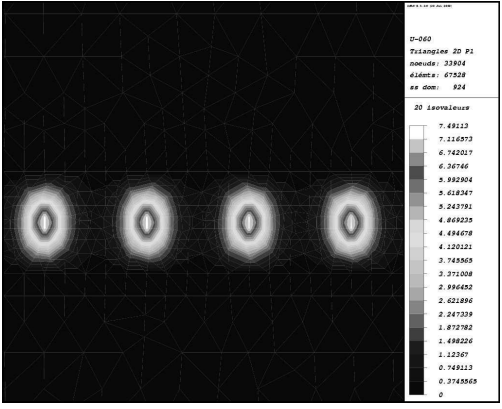

(b)

Figure 4. Small diffusion coefficient $(a=2)$. In Figure (a), isovalues of the solution of the direct problem $(t=1 / 2)$. In Figure (b), Close-up of the solution of the direct problem $(t=1 / 2)$.

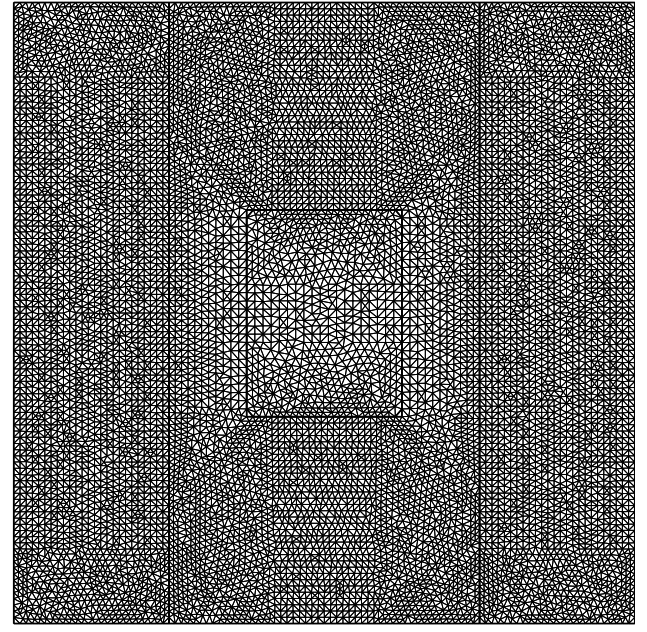

Figure 5. Mesh ( $\simeq 21000$ degrees of freedom) of the unit cell containing the waste.

Outside the repository, the asymptotic solution overestimates the concentration found in the numerical computation of the direct problem. 

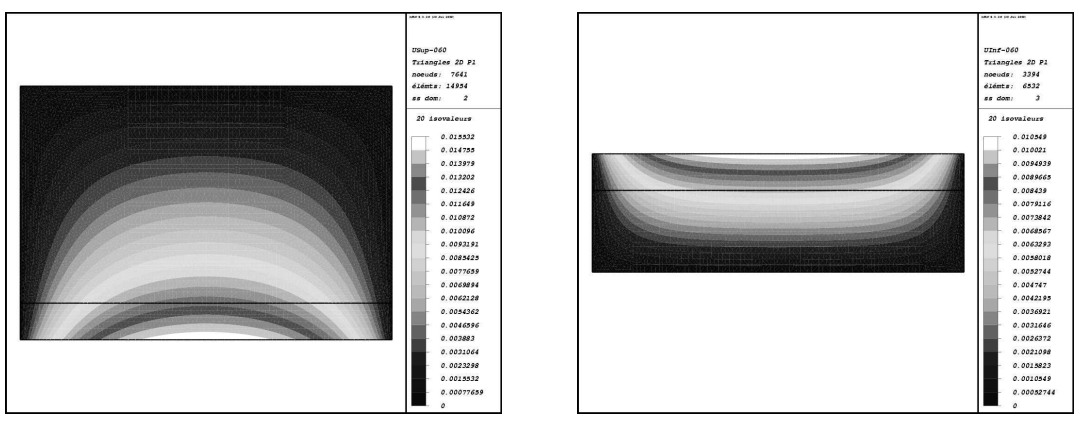

Figure 6. Small diffusion coefficient $(a=2)$ : Isovalues of the solution of the asymptotic problem (6) in the two parts of the computational domain $(t=1 / 2)$.

6.4.2. The case of large diffusion coefficient: $a=-1$. In this case, the asymptotic model features a diffusive transmission condition on the asymptotic limit surface occupied by the storage zone.

The numerical simulation of the direct problem (1) shows isovalues (Figure 8(a)) in the shape of a "smiling configuration". The same shape (Figure 8(b)) is also found in the numerical simulation of the homogenized problem (9). This numerical approximation $(\simeq 11000$ degrees of freedom) requires the knowledge of the effective coefficient $\mu^{*}$ defined by (12). Since we choose constant coefficients in the unit cell, the cell solutions $\varphi_{i}$, defined by (13), are explicit. More precisely, the horizontal solution is $\varphi_{1} \equiv 0$ and the vertical solution is $\varphi_{2}=-y_{2}$. This implies that $\mu^{*}$ is just equal to the constant value of $\mu_{1}$. The cross sections of the two solutions are displayed on Figure 9 which shows that both solutions behave similarly.

Remark 11. It is worth mentioning that the retardation factors as well as the effective porosity inside the thin strip containing the repository do not play any part in the homogenized problem (10).

Acknowledgments. This work has been supported by the Groupement de Recherches MoMaS sponsored by ANDRA, BRGM, CEA, CNRS, EDF, and IRSN. G. Allaire is a member of the DEFI project at INRIA Saclay Ile de France.

\section{REFERENCES}

[1] Dossier 2005 Argile - Évolution phénoménologique du stockage géologique, Collection Les Rapports, Juin 2005. ANDRA Agence nationale pour la gestion des déchets radioactifs.

[2] G. Allaire, Homogenization and two-scale convergence, SIAM J. Math. Anal., 23 (1992), pp. $1482-1518$. 


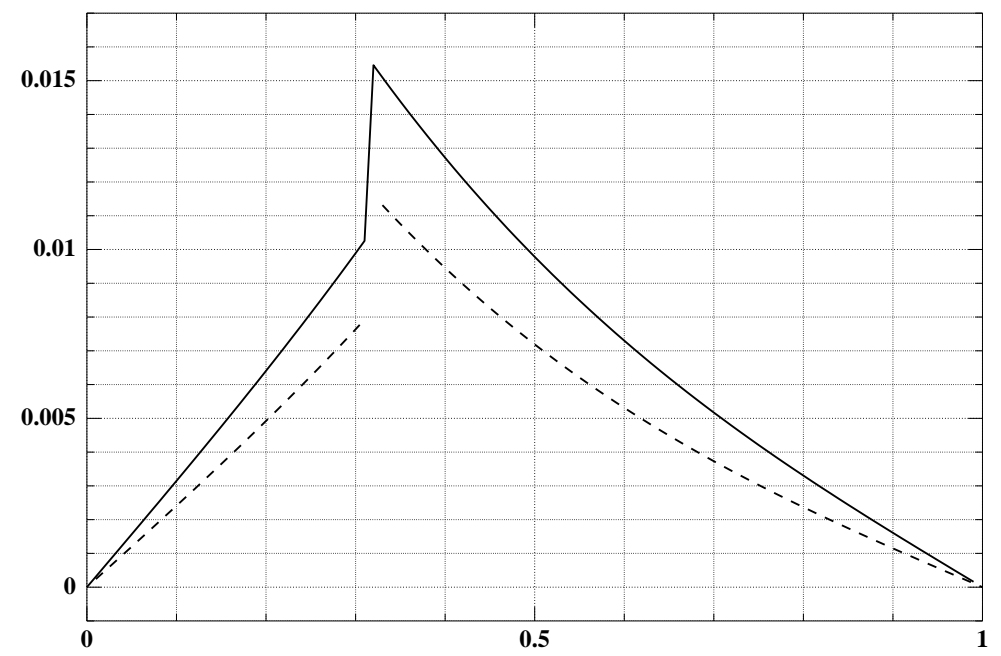

Figure 7. Small diffusion coefficient $(a=2)$ : Cross sections of the truncated (dashed) direct and asymptotic solutions at $x=0.5$ $(t=1 / 2)$.

[3] G. Allaire And C. Conca, Boundary layers in the homogenization of a spectral problem in fluid-solid structures, SIAM J. Math. Anal., 29 (1998), pp. 343-379.

[4] A. Bourgeat, O. Gipouloux, and E. Marusic-Paloka, Mathematical modeling of an array of underground waste containers, C. R. Mécanique, 330 (2002), pp. 371-376.

[5] A. Bourgeat, O. Gipouloux and E. Marusic-Paloka, Modelling of an underground waste disposal site by upscaling, Math. Methods Appl. Sci., 27(4) (2004), pp. 381-403. 


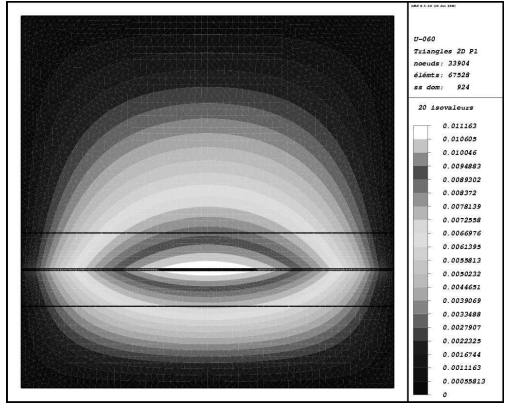

(a)

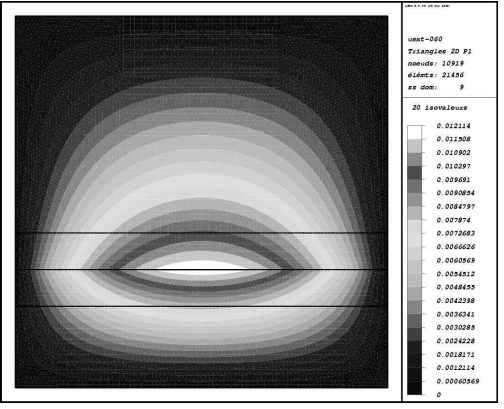

(b)

Figure 8. Large diffusion coefficient $(a=-1)$. In Figure (a) Isovalues of the solution of the direct problem $(t=1 / 2)$. In Figure (b) Isovalues of the homogenized solution (9) at $t=1 / 2$.

[6] A. Bourgeat and E. Marusic-Paloka, A homogenized model of an underground waste repository including a disturbed zone, SIAM J. Multiscale Model. Simul. 3(4) (2005), pp. 918939.

[7] A. Bourgeat, I. Boursier and D. Tromeur-Dervout, Modelling of an underground waste disposal site by upscaling, simulation with domain decomposition method, in Domain Decomposition methods in Science and Engineering, R. Kornhuber, R. Hoppe, J. Periaux, O. Pironneau, O. Widlund and J. Xu, Eds., Lecture Notes in Computational Science and Engineering, Vol 40, pp. 521-528, Springer (2004).

[8] A. Bourgeat, M. Kern, S. Schumacher, J. Talandier The COUPLEX Test Cases: Nuclear Waste Disposal Simulation, Computational Geosciences, Vol. 8, No. 2, (2004).

[9] S. Del Pino and O. Pironneau, Asymptotic analysis and layer decomposition for the couplex exercise, Computational Science 8, (2004), pp. 149-162.

[10] FreeFem++, version2.13.0, http://www.freefem.org/

[11] D. Gilbarg and N. S. Trudinger, Elliptic Partial Differential Equations of Second Order, Comprehensive Studies in Mathematics, Springer-Verlag, 2nd ed., (1983).

[12] H. P. Huy and E. Sánchez-Palencia, Phénomènes de transmission à travers des couches minces de conductivité élevée, J. Math. Anal. Appl., 47 (1974), pp. 284-309.

[13] G. NGuetseng, A general convergence result for a functional related to the theory of homogenization, SIAM J. Math. Anal. 20(3), (1989), pp. 608-623.

[14] M. Neuss-RAdU AND W. JÄGER, Effective transmission conditions for reaction diffusion processes in domains separated by an interface, SIAM J. Math. Anal., 39 (2007), pp. 687720.

[15] Test cases definition: Homogenization of the source terms, Momas Document (2007). 


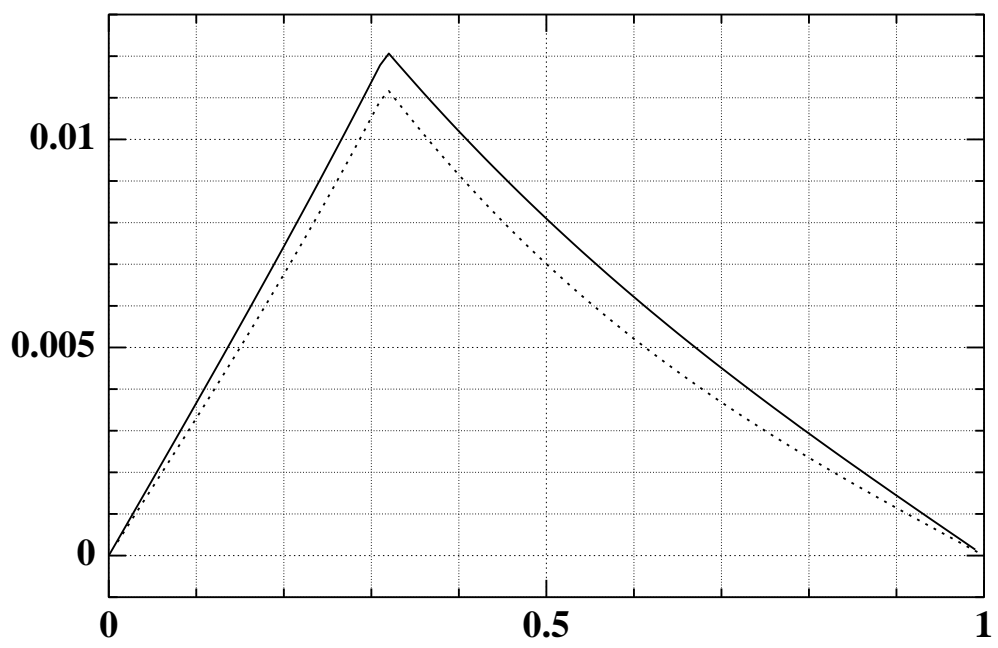

Figure 9. Large diffusion coefficient $(a=-1)$ : Cross sections of the direct versus homogenized (dashed) solutions at $x=0.5(t=$ $1 / 2)$. 